\title{
Suppression of friction-induced-vibration in MDoF systems using tangential harmonic excitation
}

\author{
Ningyu Liu $\cdot$ Huajiang Ouyang $\mathbb{D}$
}

Received: 21 January 2020/Accepted: 5 May 2020/Published online: 21 May 2020

(C) The Author(s) 2020

\begin{abstract}
This paper investigates the effects of tangential harmonic excitation on the friction-induced-vibration in multi-degree-of-freedom systems that are coupled in the tangential and normal directions. A minimal two-degree-of-freedom system and a more complicated slider-on-disc system are considered. It is observed the friction-induced-vibration of the systems can be suppressed with the tangential harmonic excitation when the amplitude and frequency of the excitation are in certain ranges. The analytical method to determine the ranges where the systems are stabilized by the tangential excitation is established. To verify the analytical results, a great amount of computational effort is also made to simulate the time responses of systems in various combinations of values of the amplitude and frequency, by which the parameter ranges where the friction-induced vibration is suppressed can also be obtained. This research can provide theoretical guidance for the suppression of friction-induced-vibration in a real disc brake system by application of a tangential harmonic excitation.
\end{abstract}

N. Liu $\cdot$ H. Ouyang $(\bowtie)$

School of Engineering, University of Liverpool, The Quadrangle, Liverpool L69 3GH, UK

e-mail: h.ouyang@liverpool.ac.uk

H. Ouyang

The State Key Laboratory for Structural Analysis of Industrial Equipment, Dalian University of Technology, Dalian 116023, China
Keywords Friction-induced-vibration · Multidegree-of-freedom system · High-frequency tangential harmonic excitation $\cdot$ Suppression

\section{Introduction}

Friction-induced-vibration exists widely in engineering applications as well as in everyday life. Examples include string music instruments, squeaking joints of robots, some insect sounds, vibration of drill strings, earthquake and automobile brake noise, etc. [1, 2]. Among them, brake noise, especially brake squeal, is still a major issue facing car manufactures today, which may cause discomfort to passengers and be perceived as quality problems, thereby increasing the warranty costs [3].

There have been a large number of published studies on friction-induced vibration. Four main mechanisms were proposed to explain the occurrence of friction-induced self-excited vibration, which are: the negative slope of the friction force-relative sliding velocity relation [4], the stick-slip vibration [5], the sprag-slip motion [6] and the mode-coupling instability [7]. The negative slope of the friction force-relative sliding velocity relation acted as negative damping in the system to cause the static equilibrium to be unstable. Popp et al. [2, 5, 8] studied discrete and continuous models exhibiting a stick-slip 
phenomenon, and rich bifurcation and chaotic behaviours were observed for a 1-DoF (degree-of-freedom) system under a harmonic excitation and multi-DoF systems. Papangelo [9] investigated the subcritical bifurcation of a slider-on-belt system which experienced friction-induced vibration in the case of a weakening-strengthening friction law, and the results showed that there was a range of the belt velocity where two stable solutions coexisted, i.e., a stable sliding equilibrium and a stable stick-slip limit cycle. Tonazzi et al. [10] performed an experimental and numerical analysis of frictional contact scenarios from macro stick-slip to continuous sliding. The stick-slip torsional vibration of a drilling system was studied in [11]. The work in [12] examined the dynamics of sprag-slip instability in a tilted-beam-on-belt system and found parameter combinations for the occurrence of sprag-slip oscillation. Sinou et al. [13] studied the instability in a nonlinear sprag-slip model with constant coefficient of friction by a central manifold theory and the effects of parameters on the sprag-slip instability were examined. The mode-coupling instability happens as some modes of the system are destabilized when coupling with other modes due to a friction-induced cross-coupling force. Hoffmann et al. $[14,15]$ investigated the physical mechanisms underlying the mode-coupling instability of self-excited friction-induced vibration and the effect of viscous damping on the mode-coupling instability, respectively. Kang et al. [16] studied the unstable vibration of a thin circular plate with friction interface and established the formulation of modal instability due to the mode-coupling of the transverse doublet modes.

In addition to the aforementioned four main mechanisms, there were other friction-related factors responsible for exciting vibration and noise. Kinkaid et al. [17] studied the dynamics of a 4-DoF (degree-offreedom) system with a two-dimension friction force and found the change of direction of the friction force could excite unstable vibration even with the Coulomb's law of friction. Chan et al. [18] analysed the destabilizing effect of the friction force modelled as a follower force. Hochlenert et al. [19] established an accurate formulation of the kinematics of the frictional contact in two and three dimensions and worked out the essential properties of the contact kinematics leading to self-excited vibration. Ouyang and Mottershead [20] investigated the instability of the transverse vibration of a disc excited by two co-rotating sliders on either side and found that the moving normal forces and the friction couple produced by the resulting friction forces brought about dynamic instability. Liu and Ouyang [21] studied the friction-induced-vibration of a slider on an elastic disc spinning at variable speeds and observed that the time-variant disc speed could be a cause for unstable vibration. Chen et al. [22] analysed the instability of a friction system caused by the time delay between the normal force and the friction force. Besides, friction-induced vibration and noise in mechanical systems were also investigated experimentally [23-27].

As with the issue of brake squeal, friction-inducedvibration in mechanical system are usually undesirable and should be avoided. Understanding the factors leading to friction-induced dynamic instability may help to select appropriate parameter values corresponding to stable systems in a design. Additionally, some structural modification approaches were developed, such as installing damping shims on the brake pads [28], modifying brake disc surface topography [29, 30], applying yawing angular misalignment [31], etc. All these methods mentioned above change the dynamic properties of the systems permanently. On the other hand, Cunefare and Graf [32] proposed using an actuator to produce a fluctuating friction force with high frequency between the pad and the disc to eliminate brake squeal. Feeny and Moon [33] applied a high-frequency excitation to quench stick-slip chaos. Zhao et al. [34] integrated the piezoceramic actuators into a disc brake system to provide harmonic high-frequency vibrations to eliminate the stick-slip limit cycle vibration of the system. This approach of applying an external periodic excitation offers a more flexible way to suppress the unwanted friction-induce vibration in mechanical systems. However, there has been little theoretical study on this topic. It was observed in [35] that sufficiently strong high-frequency excitation in the tangential direction can smoothen the discontinuity in dry friction and produce behaviour like viscous damping. Thomsen [36] conduced a theoretical analysis of the effects of highfrequency external excitation on the stick-slip vibration of a single-DoF mass-on-belt system. Nevertheless, a real mechanical systems such as a disc brake system usually involve multiple degrees of freedom, in which mechanisms responsible for friction-inducedvibration such as mode-coupling instability may appear. In order to provide more practical theoretical 
guidance for suppression of friction-induced-vibration in real disc brake systems, this paper investigates the effects of the tangential high-frequency harmonic excitation on the friction-induced dynamics of a twodegree-of-freedom mass-on-belt system and a slideron-disc system.

The rest of the paper is arranged as follows. In Sect. 2, the equations of motion are formulated and the analytical formulas for the parameter ranges where the systems are stabilized by the tangential excitation are derived for the two-DoF mass-on-belt system. In Sect. 3, the corresponding formulation and derivation for the slider-on-disc system are presented. Subsequently a detailed numerical study is conducted in Sect. 4 and the results obtained from the analytical formulas are examined in relation to the time responses calculated by the Runge-Kutta algorithm. Finally in Sect. 5 the conclusions are drawn.

\section{A minimal 2-DoF frictional system}

The model of the 2-DoF frictional system is shown in Fig. 1, which was previously investigated in [14] in terms of mode-coupling instability. In this model, a point mass $m$ is connected to the base by two sets of spring-damper systems $\left(k_{1}, c_{1}\right.$ and $\left.k_{2}, c_{2}\right)$ at the angles of inclination to the horizontal direction $\alpha_{1}$ and $\alpha_{2}$, respectively. The mass is pressed by a preload $F$ to bring it into frictional contact with a belt moving at constant velocity $v$. A spring $k_{3}$ is used to model the contact stiffness between the mass and the belt. A tangential harmonic force $F_{\mathrm{b}}(\mathrm{t})=A_{\mathrm{b}} \sin \omega t$ is now applied to the mass and its effects on the dynamic instability of the system is examined.

The equations of motion of the 2-DoF system can be derived as

$$
\begin{aligned}
& {\left[\begin{array}{cc}
m & 0 \\
0 & m
\end{array}\right]\left[\begin{array}{c}
\ddot{x} \\
\ddot{y}
\end{array}\right]+\left[\begin{array}{ll}
c_{11} & c_{12} \\
c_{21} & c_{22}
\end{array}\right]\left[\begin{array}{l}
\dot{x} \\
\dot{y}
\end{array}\right]+\left[\begin{array}{ll}
k_{11} & k_{12} \\
k_{21} & k_{22}
\end{array}\right]\left[\begin{array}{l}
x \\
y
\end{array}\right]} \\
& =\left[\begin{array}{c}
F_{\mathrm{f}}+A_{\mathrm{b}} \sin \omega t \\
-F
\end{array}\right]
\end{aligned}
$$

where,

$$
\left.\begin{array}{l}
c_{11}=c_{1} \cos ^{2} \alpha_{1}+c_{2} \cos ^{2} \alpha_{2}, k_{11}=k_{1} \cos ^{2} \alpha_{1}+k_{2} \cos ^{2} \alpha_{2}, \\
c_{12}=c_{21}=-c_{1} \sin \alpha_{1} \cos \alpha_{1}+c_{2} \sin \alpha_{2} \cos \alpha_{2}, k_{12}=k_{21}=-k_{1} \sin \alpha_{1} \cos \alpha_{1}+k_{2} \sin \alpha_{2} \cos \alpha_{2} \\
c_{22}=c_{1} \sin ^{2} \alpha_{1}+c_{2} \sin ^{2} \alpha_{2}, k_{22}=k_{1} \sin ^{2} \alpha_{1}+k_{2} \sin ^{2} \alpha_{2}+k_{3}
\end{array}\right\}
$$

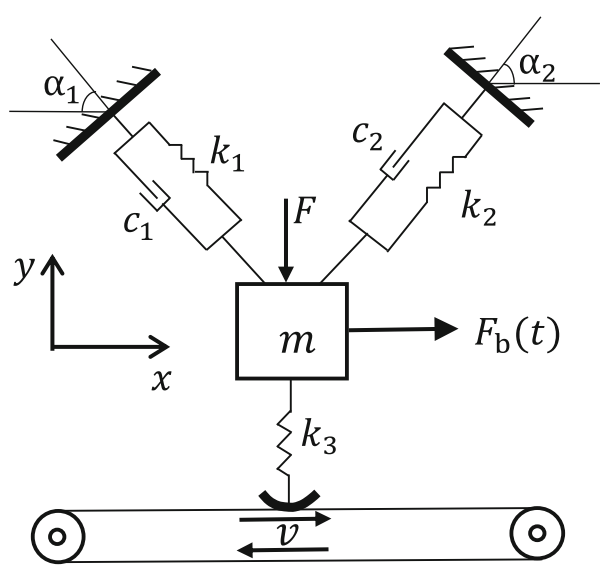

Fig. 1 Two-degree-of-freedom frictional system
And $F_{\mathrm{f}}$ represents the friction force between the mass and the belt, $F_{\mathrm{f}}=\mu\left(v_{\mathrm{r}}\right) N$, in which $\mu\left(v_{\mathrm{r}}\right)$ and $N$ denote the coefficient of friction and the normal force, respectively. Here a Coulomb's law of friction is utilized, i.e.,

$\mu\left(v_{\mathrm{r}}\right)=\operatorname{sign}\left(v_{\mathrm{r}}\right) \mu_{\mathrm{k}}$

where $v_{\mathrm{r}}=v-\dot{x}$. Equation (3) is valid during relative sliding when $v_{\mathrm{r}} \neq 0$. In the stick phase when $v_{\mathrm{r}}=0$, friction force $F_{\mathrm{f}}$ is obtained from the equations of motion, Eq. (1). Additionally, the normal force is expressed as,

$N=-k_{3} y$

By defining the following quantities and operator, 


$$
\begin{aligned}
& \omega_{1}=\sqrt{\frac{k_{1}}{m}}, \tau=\omega_{1} t, \eta_{2}=\frac{k_{2}}{k_{1}}, \eta_{3}=\frac{k_{3}}{k_{1}}, \\
& \zeta=\frac{c_{1}}{2 m \omega_{1}}, \delta=\frac{c_{2}}{c_{1}}, B_{\mathrm{b}}=\frac{A_{\mathrm{b}}}{k_{1}}, R=\frac{\omega}{\omega_{1}},(*)^{\prime}=\frac{\mathrm{d}(*)}{\mathrm{d} \tau}
\end{aligned}
$$

Equation (1) can be rewritten as,

$$
\begin{aligned}
x^{\prime \prime} & +2 \zeta\left(\cos ^{2} \alpha_{1}+\delta \cos ^{2} \alpha_{2}\right) x^{\prime}+2 \zeta\left(-\sin \alpha_{1} \cos \alpha_{1}\right. \\
& \left.+\delta \sin \alpha_{2} \cos \alpha_{2}\right) y^{\prime}+\left(\cos ^{2} \alpha_{1}+\eta_{2} \cos ^{2} \alpha_{2}\right) x \\
& +\left(-\sin \alpha_{1} \cos \alpha_{1}+\eta_{2} \sin \alpha_{2} \cos \alpha_{2}\right) y \\
& =-\mu\left(v_{r}\right) \eta_{3} y+B_{\mathrm{b}} \sin (R \tau) \\
y^{\prime \prime} & +2 \zeta\left(-\sin \alpha_{1} \cos \alpha_{1}+\delta \sin \alpha_{2} \cos \alpha_{2}\right) x^{\prime} \\
& +2 \zeta\left(\sin ^{2} \alpha_{1}+\delta \sin ^{2} \alpha_{2}\right) y^{\prime} \\
& +\left(-\sin _{1} \cos \alpha_{1}+\eta_{2} \sin \alpha_{2} \cos \alpha_{2}\right) x \\
& +\left(\sin ^{2} \alpha_{1}+\eta_{2} \sin ^{2} \alpha_{2}+\eta_{3}\right) y=-\frac{F}{k_{1}}
\end{aligned}
$$

Suppose the solutions consist of a slowly varying component and a small-amplitude fast varying component, i.e.,

$$
\begin{aligned}
& x(\tau)=\bar{x}(\tau)+\frac{1}{R} \underline{x}(R \tau) \\
& y(\tau)=\bar{y}(\tau)+\frac{1}{R} \underline{y}(R \tau)
\end{aligned}
$$

Substituting Eq. (7) into Eq. (6) results in,

$$
\begin{aligned}
\bar{x}^{\prime \prime} & +R \frac{\mathrm{d}^{2} \underline{x}}{\mathrm{~d}(R \tau)^{2}}+2 \zeta a_{1}\left(\bar{x}^{\prime}+\frac{\mathrm{d} \underline{x}}{\mathrm{~d}(R \tau)}\right) \\
& +2 \zeta a_{2}\left(\bar{y}^{\prime}+\frac{\mathrm{d} \underline{y}}{\mathrm{~d}(R \tau)}\right)+a_{4}\left(\bar{x}+\frac{1}{R} \underline{x}\right) \\
& +\left(a_{5}+\mu\left(v_{\mathrm{r}}\right) \eta_{3}\right)\left(\bar{y}+\frac{1}{R} \underline{y}\right)-B_{\mathrm{b}} \sin (R \tau) \\
& =0 \\
\left(\bar{y}^{\prime \prime}\right. & \left.+R \frac{\mathrm{d}^{2} \underline{y}}{\mathrm{~d}(R \tau)^{2}}\right)+2 \zeta a_{2}\left(\bar{x}^{\prime}+\frac{\mathrm{d} \underline{x}}{\mathrm{~d}(R \tau)}\right) \\
& +2 \zeta a_{3}\left(\bar{y}^{\prime}+\frac{\mathrm{d} \underline{y}}{\mathrm{~d}(R \tau)}\right)+a_{5}\left(\bar{x}+\frac{1}{R} \underline{x}\right) \\
& +a_{6}\left(\bar{y}+\frac{1}{R} \underline{y}\right)+\frac{F}{k_{1}} \\
& =0
\end{aligned}
$$

$$
\begin{aligned}
& \frac{\mathrm{d}^{2} \underline{y}}{\mathrm{~d}(R \tau)^{2}}=O\left(\frac{1}{R}\right) \\
& \bar{y}^{\prime \prime}+2 \zeta a_{2}\left(\bar{x}^{\prime}+\frac{\mathrm{d} \underline{x}}{\mathrm{~d}(R \tau)}\right)+2 \zeta a_{3}\left(\bar{y}^{\prime}+\frac{\mathrm{d} \underline{y}}{\mathrm{~d}(R \tau)}\right)+a_{5} \bar{x} \\
& \quad+a_{6} \bar{y}+\frac{F}{k_{1}} \\
& \quad=O\left(\frac{1}{R}\right)
\end{aligned}
$$

Assuming $B_{\mathrm{b}}=C_{\mathrm{b}} R$ and grouping the terms on the left-hand sides of Eq. (8) into the coefficients of $R, 1, \frac{1}{R}$ lead to,

$$
\begin{aligned}
& {\left[\frac{\mathrm{d}^{2} \underline{x}}{\mathrm{~d}(R \tau)^{2}}-C_{h} \sin (R \tau)\right] R+\left[\bar{x}^{\prime \prime}+2 \zeta a_{1}\left(\bar{x}^{\prime}+\frac{\mathrm{d} \underline{x}}{\mathrm{~d}(R \tau)}\right)\right.} \\
& \left.\quad+2 \zeta a_{2}\left(\bar{y}^{\prime}+\frac{\mathrm{d} \underline{y}}{\mathrm{~d}(R \tau)}\right)+a_{4} \bar{x}+a_{5} \bar{y}+\mu\left(v_{\mathrm{r}}\right) \eta_{3} \bar{y}\right] \\
& \quad+\left[a_{4} \underline{x}+a_{5} \underline{y}+\mu\left(v_{\mathrm{r}}\right) \eta_{3} \underline{y}\right] \frac{1}{R}=0
\end{aligned}
$$

When $R \gg 1$, the following equations are resulted,

$$
\frac{\mathrm{d}^{2} \underline{x}}{\mathrm{~d}(R \tau)^{2}}=C_{\mathrm{b}} \sin (R \tau)+O\left(\frac{1}{R}\right)
$$

$$
\begin{aligned}
\bar{x}^{\prime \prime} & +2 \zeta a_{1}\left(\bar{x}^{\prime}+\frac{\mathrm{d} \underline{x}}{\mathrm{~d}(R \tau)}\right)+2 \zeta a_{2}\left(\bar{y}^{\prime}+\frac{\mathrm{d} \underline{y}}{\mathrm{~d}(R \tau)}\right)+a_{4} \bar{x} \\
& +a_{5} \bar{y}+\mu\left(v_{\mathrm{r}}\right) \eta_{3} \bar{y} \\
& =O\left(\frac{1}{R}\right)
\end{aligned}
$$

where, 
It can be derived from Eqs. (11a) and (11c) that

$\underline{x}(R \tau)=-C_{\mathrm{b}} \sin (R \tau)+C_{1} R \tau+O\left(\frac{1}{R}\right)$

$\underline{y}(R \tau)=C_{2} R \tau+O\left(\frac{1}{R}\right)$

where $C_{1}$ and $C_{2}$ are constants, and $C_{1}$ and $C_{2}$ should be zero as it is unlikely for $\underline{x}$ and $\underline{y}$ to grow infinitely with time. By substituting Eqs. (12a) and (12b) into Eqs. (11b) and (11d) and applying a fast-time-average operator $\frac{1}{2 \pi} \int_{0}^{2 \pi}(*) \mathrm{d}(R \tau)$ to Eqs. (11b) and (11d) as well as omitting the small quantity $O\left(\frac{1}{R}\right)$, the new differential equations with respect to the slowly varying components $\bar{x}(\tau)$ and $\bar{y}(\tau)$ are,

$$
\begin{aligned}
& \frac{1}{2 \pi} \int_{0}^{2 \pi} \mu\left(v-\omega_{1} \bar{x}^{\prime}+\omega_{1} C_{\mathrm{b}} \cos (R \tau)\right) \mathrm{d}(R \tau) \\
& = \begin{cases}\operatorname{sign}\left(v-\omega_{1} \bar{x}^{\prime}\right) \mu_{\mathrm{k}} & \left|v-\omega_{1} \bar{x}^{\prime}\right| \geq \omega_{1} C_{\mathrm{b}} \\
{\left[-1+\frac{2}{\pi} \arccos \left(-\frac{v-\omega_{1} \bar{x}^{\prime}}{\omega_{1} C_{\mathrm{b}}}\right)\right] \mu_{\mathrm{k}}} & \left|v-\omega_{1} \bar{x}^{\prime}\right|<\omega_{1} C_{\mathrm{b}}\end{cases}
\end{aligned}
$$

Converting Eq. (13) into the first-order differential equations and linearizing the differential equations at the equilibrium point, a Jacobian matrix with respect to $\bar{x}(\tau)$ and $\bar{y}(\tau)$ can be obtained as,

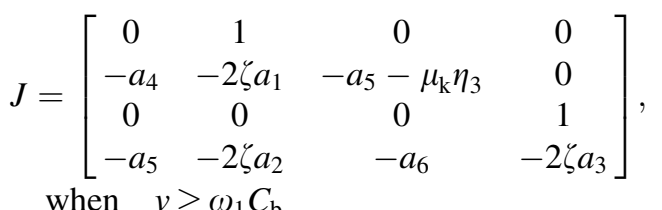

or,

$$
J=\left[\begin{array}{llll}
0 & 1 & 0 & 0 \\
-a_{4} & -2 \zeta a_{1}+\frac{2 \mu_{\mathrm{k}} \omega_{1}}{\pi \sqrt{\omega_{1}^{2} C_{\mathrm{b}}^{2}-v^{2}} \eta_{3} \bar{y}_{e}} & -a_{5}-\left[-1+\frac{2}{\pi} \arccos \left(-\frac{v}{\omega_{1} C_{\mathrm{b}}}\right)\right] \mu_{\mathrm{k}} \eta_{3} & 0 \\
0 & 0 & 0 & 1 \\
-a_{5} & -2 \zeta a_{2} & -a_{6} & -2 \zeta a_{3}
\end{array}\right], \quad \text { when } \quad v<\omega_{1} C_{\mathrm{b}}
$$

$$
\begin{aligned}
\bar{x}^{\prime \prime} & +2 \zeta a_{1} \bar{x}^{\prime}+2 \zeta a_{2} \bar{y}^{\prime}+a_{4} \bar{x}+a_{5} \bar{y} \\
& +\left[\frac{1}{2 \pi} \int_{0}^{2 \pi} \mu\left(v-\omega_{1} \bar{x}^{\prime}+\omega_{1} C_{\mathrm{b}} \cos (R \tau)\right) \mathrm{d}(R \tau)\right] \eta_{3} \bar{y} \\
& =0 \\
\bar{y}^{\prime \prime} & +2 \zeta a_{2} \bar{x}^{\prime}+2 \zeta a_{3} \bar{y}^{\prime}+a_{5} \bar{x}+a_{6} \bar{y}+\frac{F}{k_{1}}=0
\end{aligned}
$$

Because the amplitudes of the fast varying components are in the order of $\frac{1}{R}$, the behaviour of the overall system responses can be observed via the behaviour of the slowly varying components $\bar{x}(\tau)$ and $\bar{y}(\tau)$. By utilizing the friction law of Eq. (3), it is obtained that, in which $\bar{y}_{e}$ is the normal displacement of the equilibrium point obtained from the differential equation Eq. (13). The stability of the system at the equilibrium point can be then revealed by the real parts of the eigenvalues of the Jacobian matrix. The range of the amplitude and frequency of the excitation where the system is stabilized is thus derived.

\section{Slider-on-disc system}

The configuration of the slider-on-disc system is illustrated in Fig. 2. It is a simplified model of a car brake system. The brake rotor is modelled as a Kirchhoff plate clamped at inner boundary and free at outer boundary. The brake pad is modelled as a lumped mass, and the constraint to the pad imposed by the fixtures such as the calliper is modelled by a 


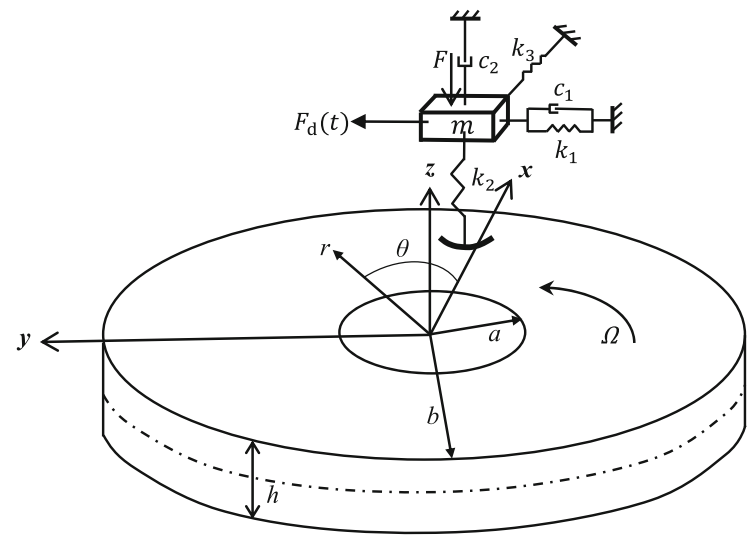

Fig. 2 The slider-on-disc system

tangential spring $k_{1}$, an inclined spring $k_{3}$ at 45 degree to the tangential direction and dashpots $c_{1}$ and $c_{2}$ in the tangential and normal directions. Without loss of generality, the circumferential coordinate of the fixtures is set as $\theta=0$. The contact stiffness between the pad and rotor is represented by a linear spring $k_{2}$. The slider is assumed to be fixed radially at $r_{0}$ form the disc centre and pressed by a preload $F$ that forces the mass into frictional contact with the disc, which is rotating at the speed $\Omega$. Likewise, a harmonic force $F_{\mathrm{d}}(t)=A_{\mathrm{d}} \sin \omega t$ in the tangential direction is applied to the slider to suppress the friction-induced vibration of the system.

In this system, the vibration of the slider in the normal and tangential directions and the transverse vibration of the disc are considered. The equations of motion of the slider can be written as,

$$
\begin{aligned}
& m r_{0} \ddot{\varphi}+c_{1} r_{0} \dot{\varphi}+k_{1} r_{0} \varphi+\frac{1}{2} k_{3} r_{0} \varphi-\frac{1}{2} k_{3} u \\
& \quad=F_{\mathrm{f}}+A_{\mathrm{d}} \sin \omega t \\
& m \ddot{u}+c_{2} \dot{u}+k_{2}\left(u-w\left(r_{0}, \varphi, t\right)\right)-\frac{1}{2} k_{3} r_{0} \varphi+\frac{1}{2} k_{3} u \\
& \quad=-F
\end{aligned}
$$

where $\varphi$ and $u$ represent the angular displacement in the tangential direction and the translational displacement in the normal direction of the slider, respectively. $w\left(r_{0}, \varphi, t\right)$ denotes the transverse displacement of the disc at the polar coordinate $\left(r_{0}, \varphi\right)$ in the space-fixed coordinate system, which is the location of the contact point at an arbitrary time $t . F_{\mathrm{f}}$ represents the friction force between the slider and the disc, $F_{\mathrm{f}}=\mu\left(v_{\mathrm{r}}\right) N$, where $\mu\left(v_{\mathrm{r}}\right)$ and $N$ denote the coefficient of friction and the normal force, respectively. In many cases the friction force decreases with the increase in the relative velocity at low velocities, therefore the friction law with a negative friction-velocity slope [37] is considered here, i.e.,

$\mu\left(v_{\mathrm{r}}\right)=\operatorname{sign}\left(v_{\mathrm{r}}\right)\left[\mu_{\mathrm{k}}+\left(\mu_{\mathrm{s}}-\mu_{\mathrm{k}}\right) \mathrm{e}^{-\alpha v_{\mathrm{r}}}\right]$

where $v_{\mathrm{r}}=r_{0}(\Omega-\dot{\varphi}), \mu_{\mathrm{s}}$ and $\mu_{\mathrm{k}}$ are the coefficients of static and kinetic friction respectively. $\alpha$ determines the initial negative slope, and the friction law actually turns into the Coulomb law when $\alpha=0$. And the normal force is expressed as,

$N=k_{2}\left(w\left(r_{0}, \varphi, t\right)-u\right)$

The transverse displacement of the disc can be approximated by a linear superposition of a set of orthogonal basis functions as [38],

$w(r, \theta, t)=\sum_{k=0}^{\infty} \sum_{l=0}^{\infty} R_{k l}(r)\left[\cos (l \theta) \cdot C_{k l}(t)+\sin (l \theta) \cdot D_{k l}(t)\right]$

where $k$ and $l$ denote the numbers of nodal circles and nodal diameters respectively, $C_{k l}(t), D_{k l}(t)$ are modal coordinates, $R_{k l}(r)$ is a combination of Bessel functions satisfying the inner and outer boundary conditions of the nonrotating disc and orthogonality conditions. The equations of motion with respect to the modal coordinates can be obtained from Lagrange's equations,

$$
\begin{aligned}
& \frac{\mathrm{d}}{\mathrm{d} t}\left[\frac{\partial L}{\partial \dot{C}_{k l}}\right]-\frac{\partial L}{\partial C_{k l}}=P_{k l}, \quad k=0,1,2, \ldots, \infty, \\
& \quad l=0,1,2, \ldots, \infty \\
& \frac{\mathrm{d}}{\mathrm{d} t}\left[\frac{\partial L}{\partial \dot{D}_{k l}}\right]-\frac{\partial L}{\partial D_{k l}}=Q_{k l}, \quad k=0,1,2, \ldots, \infty, \\
& \quad l=1,2, \ldots, \infty
\end{aligned}
$$

in which,

$L=T-U$ 
$T=\frac{1}{2} \rho h \iint_{A}\left(\frac{\partial w(r, \theta, t)}{\partial t}+\Omega \frac{\partial w(r, \theta, t)}{\partial \theta}\right)^{2} r \mathrm{~d} r \mathrm{~d} \theta$

$U=\frac{1}{2} D \iint_{A}\left(\left(\nabla^{2} w\right)^{2}-2(1-v)\right.$

$\left.\left[\frac{\partial^{2} w}{\partial r^{2}}\left(\frac{1}{r} \frac{\partial w}{\partial r}+\frac{1}{r^{2}} \frac{\partial^{2} w}{\partial \theta^{2}}\right)-\left(\frac{1}{r} \frac{\partial^{2} w}{\partial r \partial \theta}-\frac{1}{r^{2}} \frac{\partial w}{\partial \theta}\right)^{2}\right]\right) r \mathrm{~d} r \mathrm{~d} \theta$

$P_{k l}=-N \cdot \frac{\partial w\left(r_{0}, \varphi, t\right)}{\partial C_{k l}}+M_{\theta} \frac{\partial \gamma}{\partial C_{k l}}$

$Q_{k l}=-N \cdot \frac{\partial w\left(r_{0}, \varphi, t\right)}{\partial D_{k l}}+M_{\theta} \frac{\partial \gamma}{\partial D_{k l}}$

$\gamma=\frac{\partial w\left(r_{0}, \varphi, t\right)}{r_{0} \partial \theta}$

In the above equations, $T$ and $U$ denote the kinetic energy and strain energy of the disc respectively, $P_{k l}$ and $Q_{k l}$ represent the generalized forces obtained from the virtual work of the normal contact force and bending moment acting on the disc. $A$ is the area of the disc surface, $\rho$ is the density of material, $D=\frac{E h^{3}}{12\left(1-v^{2}\right)}$ is the bending rigidity, $E$ and $v$ are the Young's modulus and the Poisson's ratio of the disc material, respectively. The bending moment $M_{\theta}$ can be expressed as,

$M_{\theta}=h F_{\mathrm{f}} / 2$

By substituting Eqs. (19), (20) and (29) into Eqs. (21-28), the equations of transverse vibration of the disc with respect to the modal coordinates can be derived,

$$
\begin{aligned}
M_{k l} \ddot{C}_{k l}+2 l M_{k l} \Omega \dot{D}_{k l}+\left(\omega_{k l}^{2} M_{k l}-l^{2} M_{k l} \Omega^{2}\right) C_{k l} \\
=\left[-R_{k l}\left(r_{0}\right) \cos (l \varphi)-\frac{h}{2 r_{0}} \mu\left(v_{\mathrm{r}}\right) l R_{k l}\left(r_{0}\right) \sin (l \varphi)\right] \\
\quad \cdot\left[k_{2}\left(\sum_{k=0}^{\infty} \sum_{l=0}^{\infty} R_{k l}\left(r_{0}\right)\left(\cos (l \varphi) \cdot C_{k l}(t)+\sin (l \varphi) \cdot D_{k l}(t)\right)-u\right)\right]
\end{aligned}
$$

$$
\begin{aligned}
M_{k l} \ddot{D}_{k l}-2 l M_{k l} \Omega \dot{C}_{k l}+\left(\omega_{k l}^{2} M_{k l}-l^{2} M_{k l} \Omega^{2}\right) D_{k l} \\
=\left[-R_{k l}\left(r_{0}\right) \sin (l \varphi)+\frac{h}{2 r_{0}} \mu\left(v_{r}\right) l R_{k l}\left(r_{0}\right) \cos (l \varphi)\right] \\
\quad \cdot\left[k_{2}\left(\sum_{k=0}^{\infty} \sum_{l=0}^{\infty} R_{k l}\left(r_{0}\right)\left(\cos (l \varphi) \cdot C_{k l}(t)+\sin (l \varphi) \cdot D_{k l}(t)\right)-u\right)\right]
\end{aligned}
$$

in which $\omega_{k l}$ is the natural frequency of the mode with $k$ nodal circles and $l$ nodal diameters of the corresponding nonrotating plate, and

$M_{k l}= \begin{cases}\rho h \pi \int_{a}^{b} R_{k l}^{2}(r) r \mathrm{~d} r, & l=1,2, \ldots \\ 2 \rho h \pi \int_{a}^{b} R_{k l}^{2}(r) r \mathrm{~d} r, & l=0\end{cases}$

The equations of motion for the whole system are therefore the coupled equations consisting of Eqs. (16), (17) and (30). Similar to the 2-DoF frictional system, the analytical formulas to determine the range of the amplitude and frequency of the excitation where the system can be stabilized are derived. The detailed derivation is given in the "Appendix".

\section{Numerical study}

In this section, a detailed numerical study is conducted to demonstrate the effect of tangential harmonic excitation in suppressing the friction-induced vibration of the 2-DoF system and the slider-on-disc system. For the determination of the parameter range that will suppress the friction-induced vibration of the systems, the results obtained both from the analytical method and from the time domain integration by the Runge-Kutta algorithm are presented. To avoid the numerical difficulty brought about by the discontinuity at the zero relative velocity of the discontinuous type of friction laws, the smooth functions $[39,40]$ that can accurately describe the behaviour of the discontinuous systems are used to approximate the discontinuous friction forces in the calculation of the time responses. In this paper, the smooth functions used are $\mu\left(v_{\mathrm{r}}\right)=\mu_{\mathrm{k}} \tanh \left(\sigma v_{\mathrm{r}}\right)$ in the 2-DoF frictional system and $\mu\left(v_{\mathrm{r}}\right)=\left[\mu_{\mathrm{k}}+\left(\mu_{\mathrm{s}}-\mu_{\mathrm{k}}\right) \mathrm{e}^{-\alpha v_{\mathrm{r}}}\right] \tanh \left(\sigma v_{\mathrm{r}}\right)$ in the slider-on-disc system, respectively. $\sigma$ is the smoothness factor and its value is set as 50 in all the time responses simulations of both systems. 

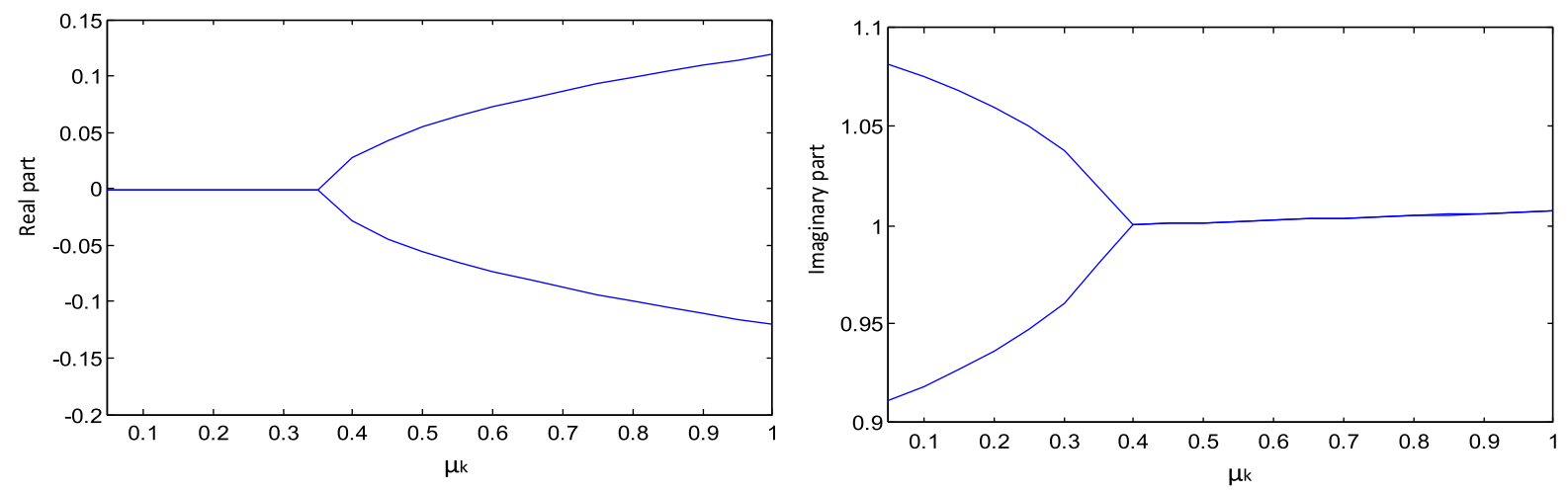

Fig. 3 The eigenvalue of the Jacobian matrix of the original system as a function of $\mu_{\mathrm{k}}$

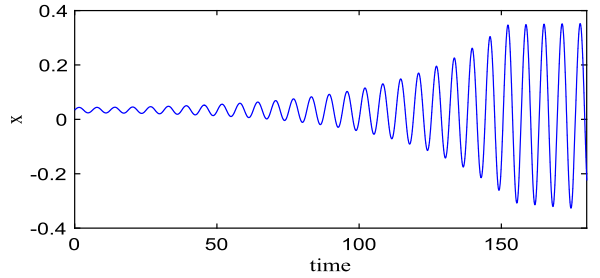

(a)

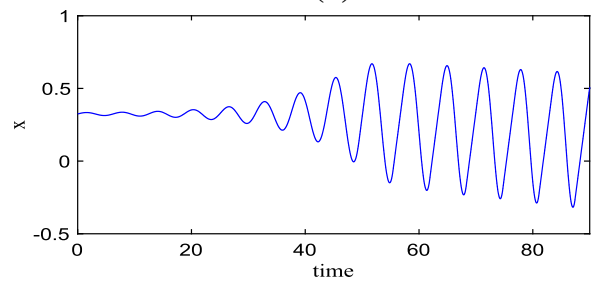

(c)

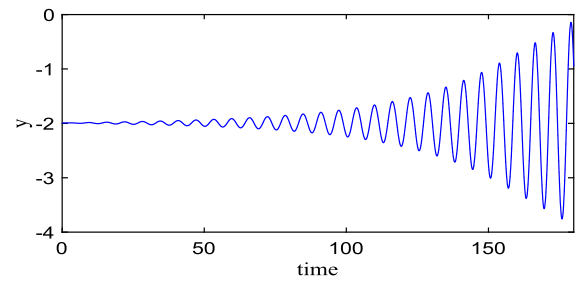

(b)

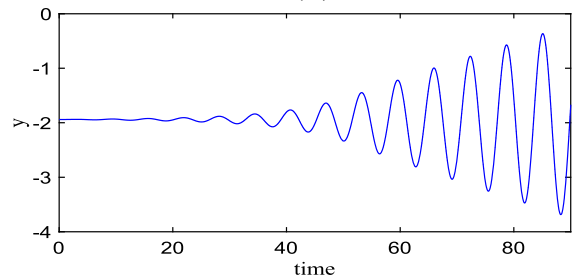

(d)

Fig. 4 The time histories of tangential and normal displacements of the original system when $\mu_{\mathrm{k}}$ is larger than its critical value: $\mathbf{a}$, b $\mu_{\mathrm{k}}=0.4 ; \mathbf{c}, \mathbf{d} \mu_{\mathrm{k}}=0.7$

\subsection{Numerical study of the 2-DoF frictional system}

In the numerical examples for the 2-DoF friction system, the basic system parameters assigned with constant values are: $m=1, \alpha_{1}=30^{\circ}, \alpha_{2}=45^{\circ}$, $k_{1}=1, k_{2}=0.5, k_{3}=0.5, c_{1}=0.001, c_{2}=0.001$, $F=2$. Firstly the dynamics of the original system, i.e. when the tangential harmonic excitation is not applied, is examined. The eigenvalues of the Jacobian matrix of the original system as a function of $\mu_{\mathrm{k}}$ are shown in Fig. 3, which reflects the local stability of the system at the equilibrium point. If there exists an eigenvalue with a positive real part, the equilibrium point is unstable and the system exhibits growing self-excited vibration. It is clearly shown that the mode-coupling instability appears at the onset of a positive real part and the merging of imaginary parts in the original frictional system. And the equilibrium point of the system becomes destabilized when $\mu_{\mathrm{k}}$ is larger than its critical value 0.37 . Figure 4 displays the time histories of the tangential and normal displacements of the original system at $\mu_{\mathrm{k}}=0.4$ and $\mu_{\mathrm{k}}=0.7$, where it can be seen that the amplitudes of the dynamic responses, especially the normal displacement of the mass, grow with time until the mass separates with the disc (when $y>0$ ). Then the mass will re-contact with and separate from the belt repetitively, as has been observed in [41]. The time histories after separation are not shown here as the present work is devoted to 
Fig. 5 The dynamic responses of the system after application of the harmonic excitation with amplitude $A_{\mathrm{b}}=35$ and frequency ratio $R=\frac{\omega}{\omega_{1}}=20: \mathbf{a}, \mathbf{b} \mu_{\mathrm{k}}=0.4$; c, $\mathbf{d} \mu_{\mathrm{k}}=0.7$. (The belt velocity is 0.3 )

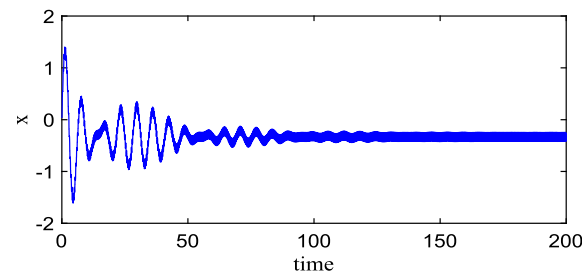

(a)

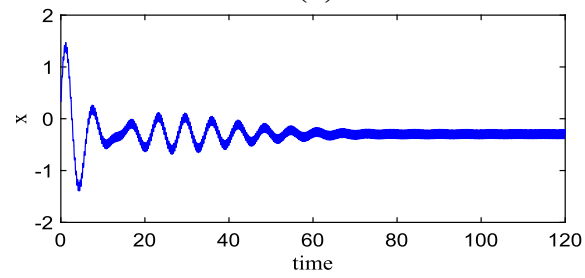

(c)

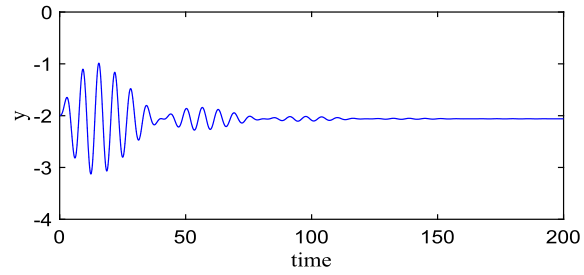

(b)

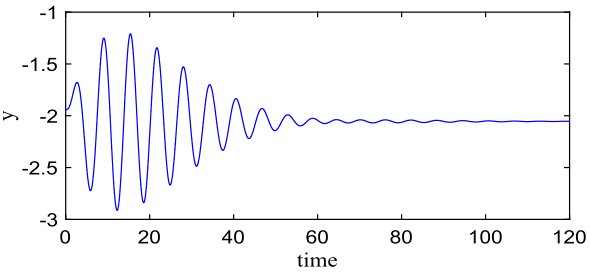

(d) suppressing the friction-induced vibration of the system by means of tangential harmonic excitation. Then the dynamic responses of the system under the above two values of $\mu_{\mathrm{k}}$ in the presence of the harmonic excitation with appropriate amplitude and frequency are shown in Fig. 5, from which it is observed that although the amplitudes of the dynamic responses are increased in the initial stage under the influence of the external excitation, they are greatly attenuated in the steady-state phase, especially for the normal displacements of the mass. These two cases demonstrate the effectiveness of the tangential harmonic excitation in suppressing the friction-induced self-excited vibration of the system.

Next the range of the amplitude and frequency of the harmonic excitation to stabilize the frictional system is derived. The results obtained from the analytical method and from the extensive time response simulations are compared, as shown in Fig. 6. In this figure, the region above each curve incorporates the parameter combinations to stabilize the system under the specific coefficient of friction (greater than the critical value). It is seen that there is fairly good agreement between the stability boundaries obtained from the analytical method and from the time response simulations when the frequency ratio ( $\left.R=\omega / \omega_{1}\right)$ is sufficiently large. Besides, it should be noted here the mode-coupling instability of the original system is not dependent on belt velocity $v$, which, however, has an effect on the stability

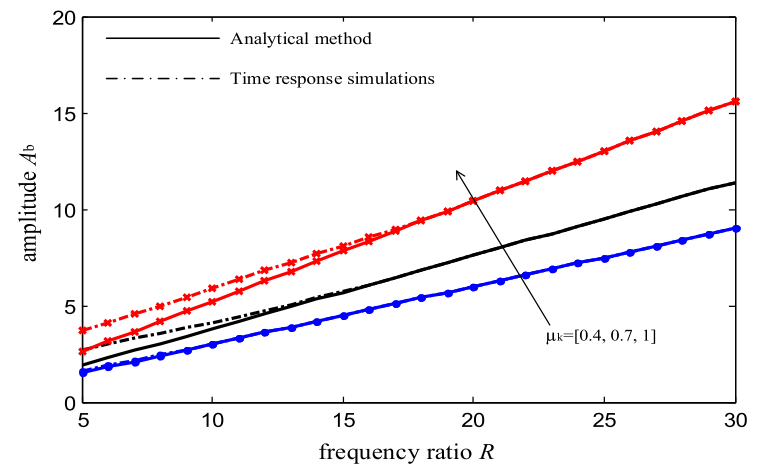

Fig. 6 The range of the amplitude and frequency of the harmonic excitation to stabilize the system obtained from both the analytical method and the extensive time response simulations. ( $v=0.3$, the parameter range to stabilize the system is above the corresponding curve)

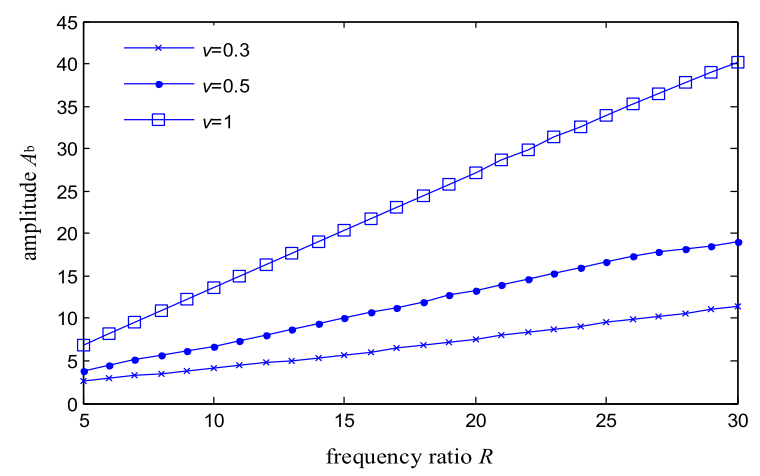

Fig. 7 The range of the amplitude and frequency of the harmonic excitation to stabilize the system under three different values of belt velocity $\left(\mu_{\mathrm{k}}=0.7\right.$, the parameter range to stabilize the system is above the corresponding curve) 
Table 1 The values of the constant system parameters

\begin{tabular}{lllllll}
\hline$a$ & $b$ & $r_{0}$ & $\rho$ & $E$ & $h$ & $v$ \\
\hline $0.044 \mathrm{~m}$ & $0.12 \mathrm{~m}$ & $0.1 \mathrm{~m}$ & $7200 \mathrm{~kg} / \mathrm{m}^{3}$ & $150 \mathrm{GPa}$ & $0.002 \mathrm{~m}$ & 0.211 \\
$m$ & $k_{1}$ & $k_{2}$ & $k_{3}$ & $c_{1}$ & $c_{1}$ & \\
$1 \mathrm{~kg}$ & $10^{5} \mathrm{~N} / \mathrm{m}$ & $5 \times 10^{4} \mathrm{~N} / \mathrm{m}$ & $6 \times 10^{4} \mathrm{~N} / \mathrm{m}$ & $5 \mathrm{~N} \mathrm{~s} / \mathrm{m}$ & $5 \mathrm{~N} \mathrm{~s} / \mathrm{m}$ & \\
\hline
\end{tabular}

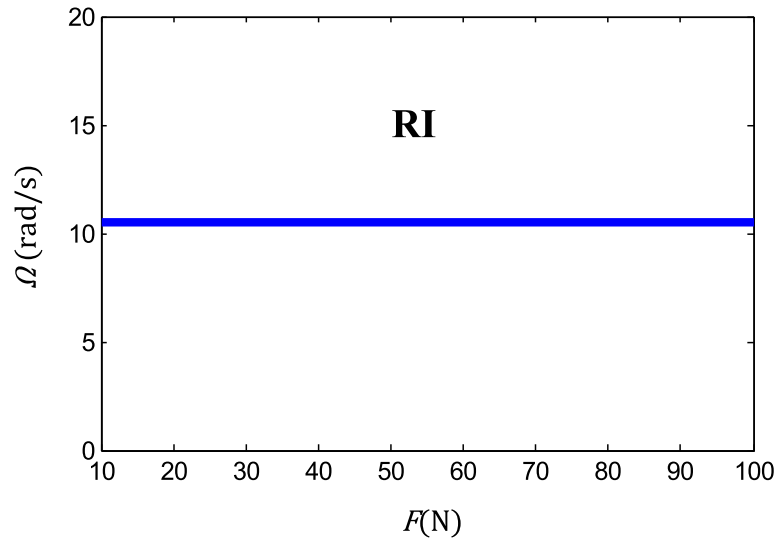

(a)

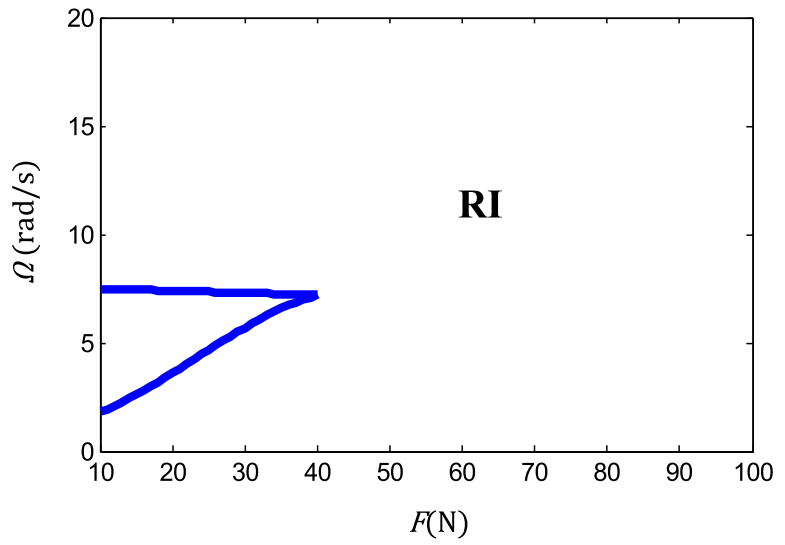

(b)

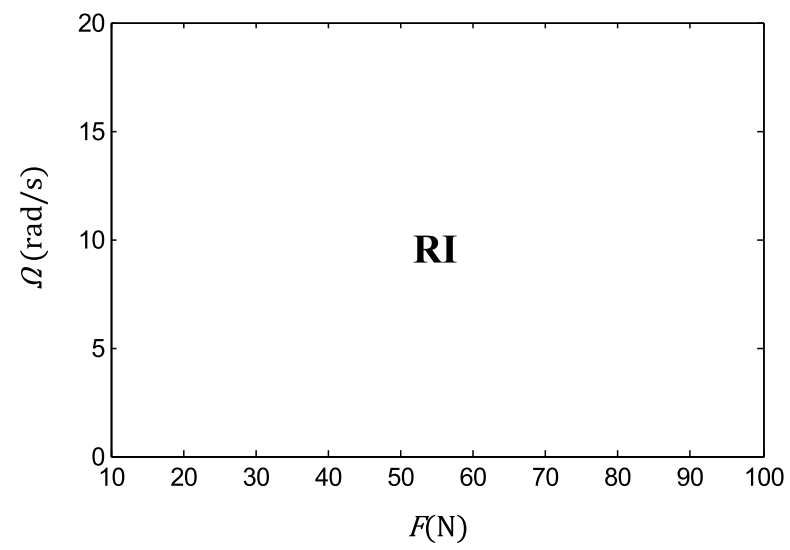

(c)

Fig. 8 The region of instability of normal preload versus disc speed in three cases of friction coefficients: $\mathbf{a} \mu_{\mathrm{s}}=1, \alpha=0, \mathbf{b} \mu_{\mathrm{s}}=1.5$, $\mu_{\mathrm{k}}=1.2, \alpha=1, \mathbf{c} \mu_{\mathrm{s}}=2.25, \mu_{\mathrm{k}}=2, \alpha=1$

boundary for the harmonic excitation. Figure 7 shows the range of the amplitude and frequency of the harmonic excitation to stabilize the system under three different values of belt velocity, from which it is observed that a larger-amplitude excitation is needed to suppress the unstable vibration of the frictional system in the situation of higher belt velocity.

\subsection{Numerical study of the slider-on-disc system}

The basic system parameters whose values are constant in the numerical examples are listed in Table 1. It should be noted that numbers $k$ and $l$ in the expression of the transverse displacement of the disc can be chosen to include as many modes as needed to represent the dynamics of the system with acceptable accuracy. To avoid excessive computations, the 


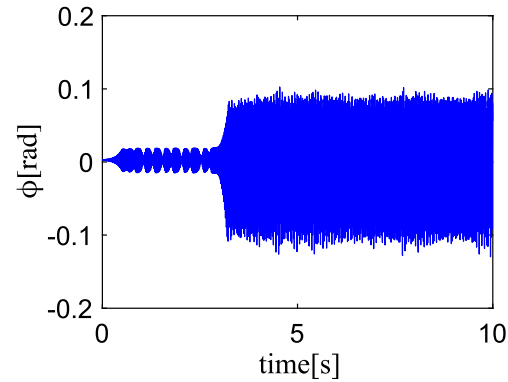

(a)

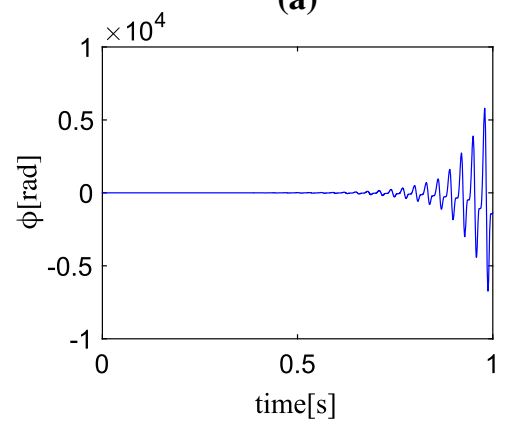

(d)

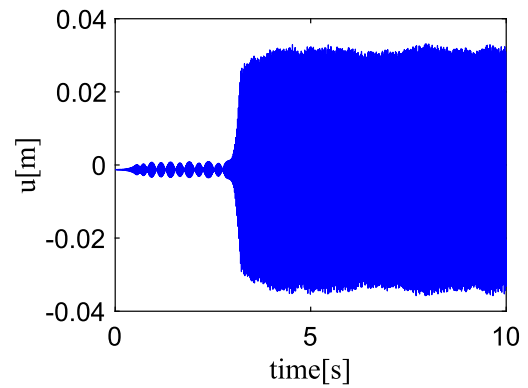

(b)

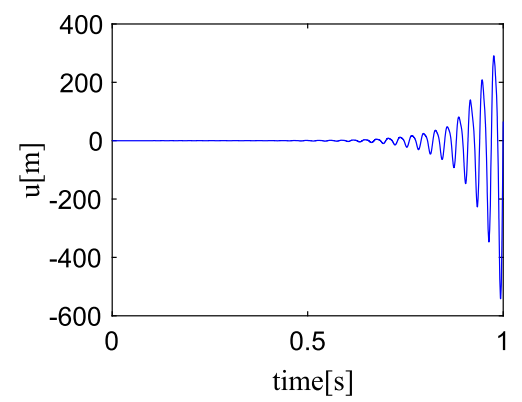

(e)

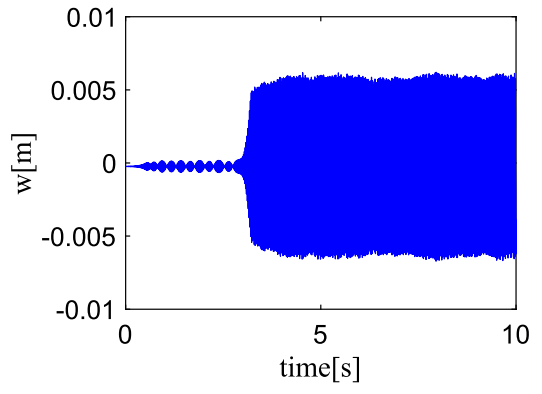

(c)

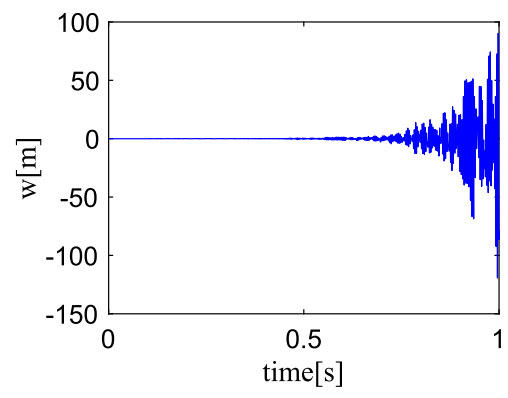

(f)
Fig. 9 The dynamic responses of system when $F=100 \mathrm{~N}$ and $\Omega=5 \mathrm{rad} / \mathrm{s}$ including the angular displacement of the slider in the tangential direction, the translational displacement of the

modal series in Eq. (20) are truncated at suitable values of $k$ and $l$. The first seven natural frequencies of the disc are 1492, 1517, 1517, 1824, 1824, 2774 and $2774 \mathrm{rad} / \mathrm{s}$ and the critical speed of the disc is $\omega_{\mathrm{cr}}=\frac{1824}{2}=912 \mathrm{rad} / \mathrm{s}$. It is found that the first seven disc modes (one single mode with zero nodal circle and zero nodal diameter and three pairs of doublet modes with zero nodal circle and one, two or three nodal diameters) are adequate in terms of the convergence of the results.

Firstly, the dynamic characteristics of the original system (when the tangential harmonic excitation is not applied) is analysed. The dynamic instability in the original system can be contributed by three factors, i.e. the negative slope in the friction force-relative velocity relationship, the mode coupling instability of the normal and tangential motion of the slider and the effect of moving load which causes speed-dependent instability, all of which the brake system may encounter in practice $[3,42]$. The local stability of the system at the sliding equilibrium can be used to evaluate if the self-excited vibration will be generated, which is studied in the following procedures. First of slider in the normal direction and the transverse displacement of the disc at the point $r=r_{0}$ and $\theta=0: \mathbf{a}-\mathbf{c} \mu_{\mathrm{s}}=1.5, \mu_{\mathrm{k}}=1.2$, $\alpha=1 ; \mathbf{d}-\mathbf{f} \mu_{\mathrm{s}}=2.25, \mu_{\mathrm{k}}=2, \alpha=1$

all, the sliding equilibrium of this system is found by solving the algebraic nonlinear equations derived from setting all the terms involving velocity and acceleration in the coupled equations Eqs. (16), (17) and (30) to be zero. In the second step, the nonlinear coupled equations governing the motion of the system are linearized at the sliding equilibrium and the Jacobian matrix is extracted from the linearized system. In the last step, the eigenvalues of the Jacobian matrix are calculated to reveal the local stability of the system at the sliding equilibrium for various values of parameters. Figure 8 plots the regions of instability (RI) dependent on the normal preload versus the disc speed under three different values of the coefficients of friction. In Fig. 8a when $\alpha=0$, the coefficients of friction are set as constant at $\mu\left(v_{\mathrm{r}}\right)=\mu_{\mathrm{S}}=1$, the dynamic instability stems from only the moving load which causes instability as disc speed $\Omega>10.5 \mathrm{rad} / \mathrm{s}$. In Fig. 8b, the coefficients of friction are not large enough to bring about the mode-coupling instability, but the large negative slope of the friction-velocity relationship in the vicinity of zero relative velocity and the moving load lead to the dynamic instability in low and high disc speeds, respectively. In Fig. 8c, the 


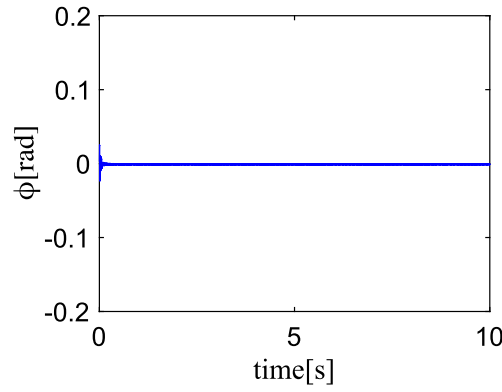

(a)

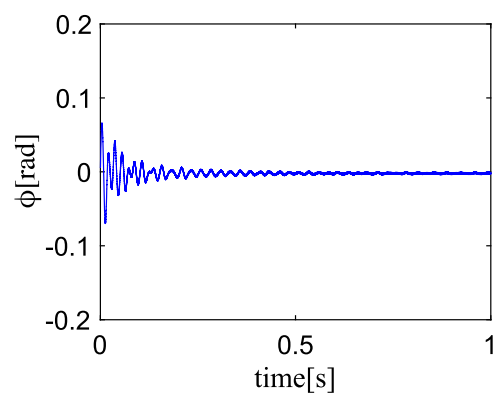

(d)

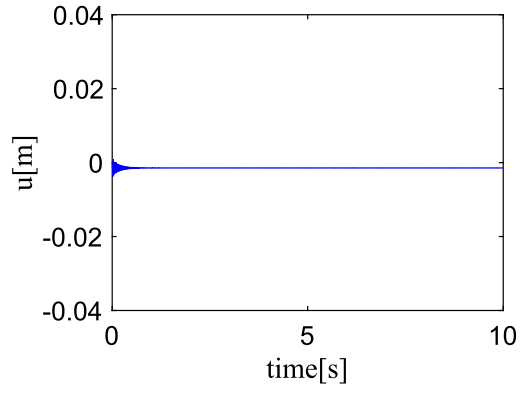

(b)

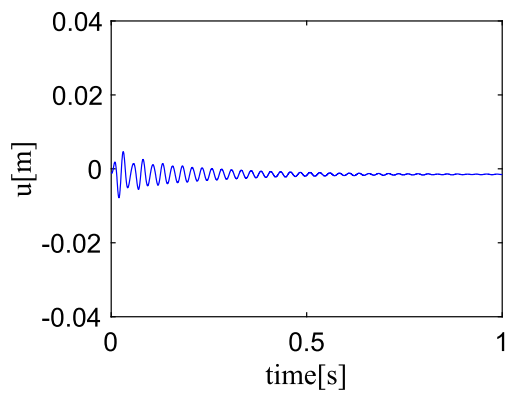

(e)

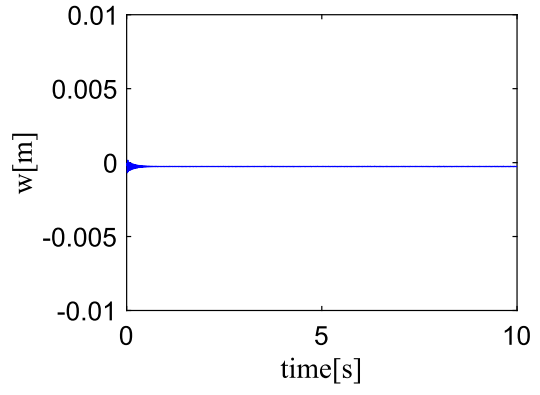

(c)

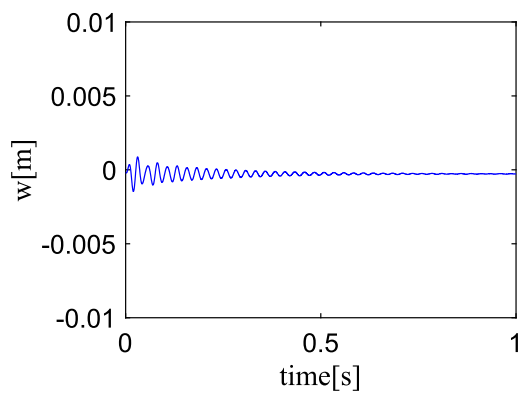

(f)

Fig. 10 The dynamic responses of system after application of the harmonic excitation for the two cases in Fig. 9: a-c for the first case with the excitation amplitude $A_{\mathrm{d}}=2 \times 10^{4} \mathrm{~N}$ and frequency ratio $R=\frac{\omega}{\omega_{\mathrm{cr}}}=22 ; \mathbf{d}-\mathbf{f}$ for the second case with the

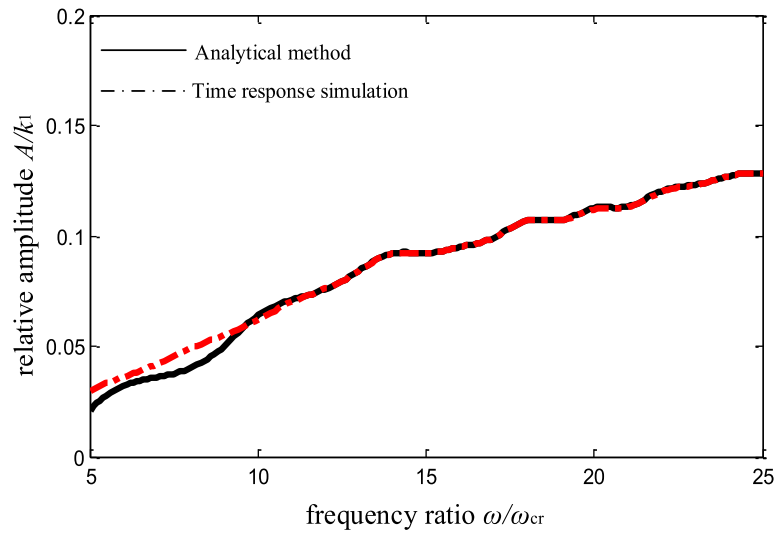

(a)

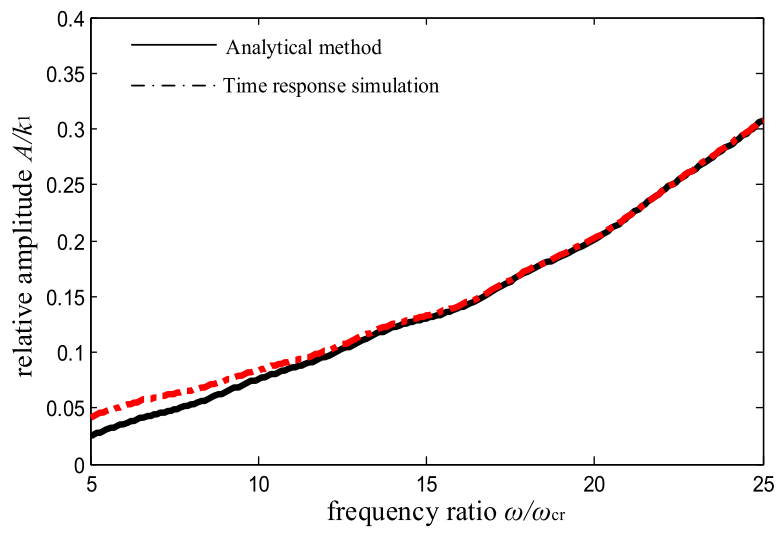

(b)

Fig. 11 The range of the amplitude and frequency of the harmonic excitation to suppress the friction-induced vibration of the system: $\mathbf{a}$ the first case $\mathbf{b}$ the second case. (The parameter range to suppress the friction-induced vibration is above the corresponding curve)

mode-coupling instability exists, therefore the dynamic instability occurs in the system independent of the values of the normal preload and disc speed. The time histories of the dynamic responses of the system with two sets of parameters whose values are in the excitation amplitude $A_{\mathrm{d}}=5 \times 10^{4} \mathrm{~N}$ and frequency ratio $R=\frac{\omega}{\omega_{\mathrm{cr}}}=22$ region of instability are displayed in Fig. 9, where the friction-induced self-excited vibration is clearly exhibited in the two cases. It should be noted that since the interest here is only to identify the occurrence of friction-induced vibration in the original 


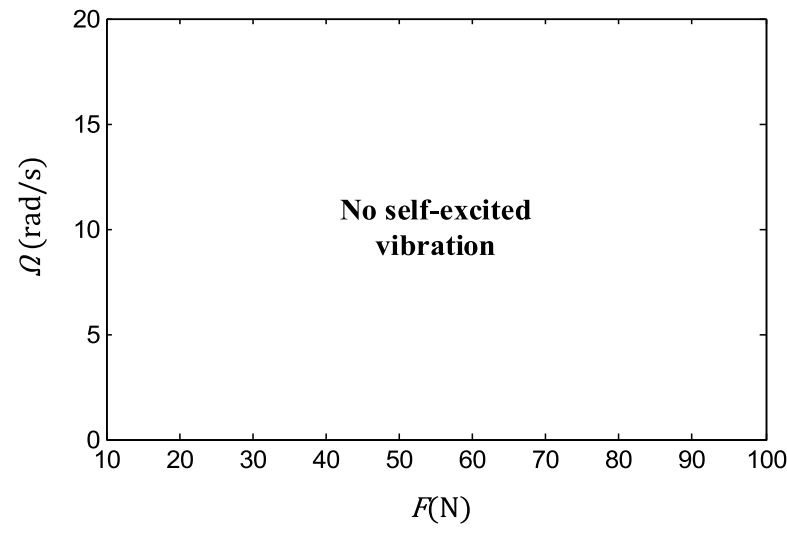

(a)

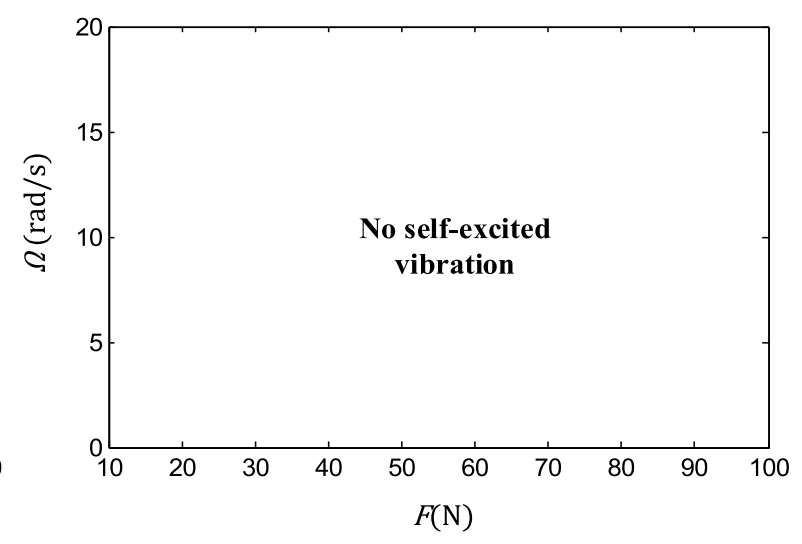

(b)

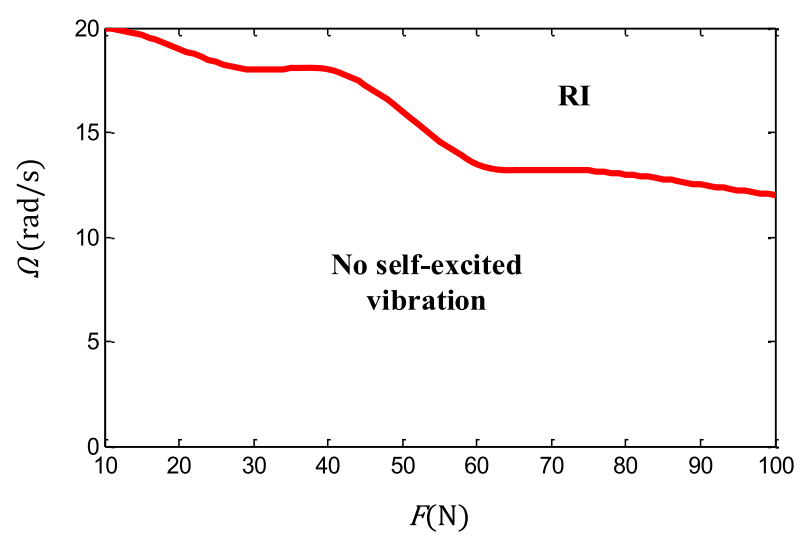

(c)

Fig. 12 The region of instability in three cases of friction coefficients in application of the harmonic excitation with the amplitude $A_{\mathrm{d}}=5 \times 10^{4} \mathrm{~N}$ and frequency ratio $R=20: \mathbf{a} \mu_{\mathrm{s}}=1, \alpha=0, \mathbf{b} \mu_{\mathrm{s}}=1.5, \mu_{\mathrm{k}}=1.2, \alpha=1, \mathbf{c} \mu_{\mathrm{s}}=2.25, \mu_{\mathrm{k}}=2, \alpha=1$

system, the behaviour of separation and re-contact between the slider and the disc is not considered, which thus allows the dynamic responses to grow boundlessly. Then the dynamic responses of the system for the two cases after application of the tangential harmonic excitation with appropriate amplitude and frequency are shown in Fig. 10, which obviously indicates that the friction-induced vibration in the original system is suppressed by the highfrequency tangential harmonic excitation.

Next the ranges of the amplitude and frequency of the harmonic excitation to suppress the frictioninduced vibration of the system are derived, when the values of system parameters are identical to those in Fig. 9. Both the results obtained from the analytical method and from extensive time responses simulations are shown for the first case in Fig. 11a and second case in Fig. 10b, where good agreements between the results from the two approaches are observed for the two cases of system when the frequency ratio is sufficiently large. It can therefore be concluded that the analytical method is a reliable approach, while the time responses simulations can also be performed for verification in the practical applications. Besides, the regions of instability of the original system shown in Fig. 8 can be greatly diminished by application of the tangential harmonic excitation with appropriate amplitude and frequency, as depicted in Fig. 12. Consequently, the application of the tangential harmonic excitation with appropriate amplitude and frequency on the brake pad serves as an effective way to help develop quieter brakes, i.e. lower probability of occurrence of friction-induced vibration. 


\section{Conclusions}

In this paper, the friction-induced vibration of a twodegree-of-freedom mass-on-belt system and a complicated slider-on-disc system, as well as the application of the harmonic excitation in the direction tangential to the friction interface for suppressing the friction-induced vibration in the systems, are studied. In the multi-degree-of-freedom frictional systems, there is one or several friction-related factors, e.g., the mode-coupling instability, the negative slope in the friction force-relative velocity relationship, the moving load, contributing to the occurrence of self-excited vibration. The results show the tangential harmonic excitation with appropriate amplitude and frequency is very effective in suppressing the friction-induced selfexcited vibration of the systems. The ranges of the amplitude and frequency of the harmonic excitation that stabilize the friction systems are obtained by both an analytical method and extensive time response simulations. The results by the two approaches are in good agreement when the ratio between the excitation frequency and the reference frequency (associated with a natural frequency of the system and in the same order as it) is sufficiently large. This research provides theoretical guidance for applying the tangential harmonic excitation to suppress the friction-inducedvibration in real disc brake systems. In practice, piezoactuators are usually used to drive the pad into high-frequency tangential vibration to suppress squeal, which use voltage as input. Therefore, some experimental tests must be carried out to establish the correlation between the amplitudes of the external excitation in the numerical simulations and the amplitudes of the input voltage for the piezoactuators in the practical application. In the future, the FE (finite element) model of a real brake system will also be theoretically investigated and experimental tests will be carried out.

Acknowledgements The Ningyu Liu is sponsored by a University of Liverpool and China Scholarship Council joint scholarship. This research is also supported by the National Science Foundation of China (11672052).

\section{Compliance with ethical standards}

Conflict of interest The authors declare that they have no conflict of interest.
Open Access This article is licensed under a Creative Commons Attribution 4.0 International License, which permits use, sharing, adaptation, distribution and reproduction in any medium or format, as long as you give appropriate credit to the original author(s) and the source, provide a link to the Creative Commons licence, and indicate if changes were made. The images or other third party material in this article are included in the article's Creative Commons licence, unless indicated otherwise in a credit line to the material. If material is not included in the article's Creative Commons licence and your intended use is not permitted by statutory regulation or exceeds the permitted use, you will need to obtain permission directly from the copyright holder. To view a copy of this licence, visit http://creativecommons.org/licenses/by/4.0/.

\section{Appendix}

By defining the following quantities and operator,

$$
\begin{gathered}
\tau=\omega_{\mathrm{cr}} t, \omega_{1}=\sqrt{k_{1} / m}, \omega_{2}=\sqrt{k_{2} / m}, \omega_{3}=\sqrt{k_{3} / m}, \\
\beta_{i}=\frac{\omega_{i}}{\omega_{\mathrm{cr}}}(i=1,2,3), \beta_{k l}=\frac{\omega_{k l}}{\omega_{\mathrm{cr}}}, \bar{\Omega}=\frac{\Omega}{\omega_{\mathrm{cr}}}, \\
\gamma_{k l}=\frac{m}{M_{k l}}, \zeta_{i}=\frac{c_{i}}{2 \sqrt{m k_{i}}}, B_{\mathrm{d}}=\frac{A_{\mathrm{d}}}{m \omega_{\mathrm{cr}}^{2}}, R=\frac{\omega}{\omega_{\mathrm{cr}}}, \\
(*)^{\prime}=\frac{\mathrm{d}(*)}{\mathrm{d} \tau}
\end{gathered}
$$

The coupled differential equations Eqs. (16), (17) and (30) governing the motion of the slider-on-disc system can be rewritten as,

$$
\begin{aligned}
& r_{0}\left(\varphi^{\prime \prime}+2 \zeta_{1} \beta_{1} \varphi^{\prime}+\beta_{1}^{2} \varphi+\frac{1}{2} \beta_{3}^{2} \varphi\right)-\frac{1}{2} \beta_{3}^{2} u \\
& =\mu\left(v_{\mathrm{r}}\right) \beta_{2}^{2}(w-u)+B_{\mathrm{d}} \sin (R \tau) \\
& u^{\prime \prime}+2 \zeta_{2} \beta_{2} u^{\prime}+\beta_{2}^{2}(u-w)-\frac{1}{2} \beta_{3}^{2} r_{0} \varphi+\frac{1}{2} \beta_{3}^{2} u=\frac{-F}{m \omega_{\mathrm{cr}}^{2}} \\
& C_{k l}^{\prime \prime}+2 l \bar{\Omega} D_{k l}^{\prime}+\left(\beta_{k l}^{2}-l^{2} \bar{\Omega}^{2}\right) C_{k l} \\
& =\gamma_{k l} \beta_{2}^{2}\left[-R_{k l}\left(r_{0}\right) \cos (l \varphi)-\frac{h}{2 r_{0}} \mu\left(v_{\mathrm{r}}\right) l R_{k l}\left(r_{0}\right) \sin (l \varphi)\right] \\
& \quad\left(\sum_{k=0}^{\infty} \sum_{l=0}^{\infty} R_{k l}\left(r_{0}\right)\left[\cos (l \varphi) \cdot C_{k l}+\sin (l \varphi) \cdot D_{k l}\right]-u\right)
\end{aligned}
$$




$$
\begin{aligned}
D_{k l}^{\prime \prime} & -2 l \bar{\Omega} C_{k l}^{\prime}+\left(\beta_{k l}^{2}-l^{2} \bar{\Omega}^{2}\right) D_{k l} \\
& =\gamma_{k l} \beta_{2}^{2}\left[-R_{k l}\left(r_{0}\right) \sin (l \varphi)+\frac{h}{2 r_{0}} \mu\left(v_{\mathrm{r}}\right) l R_{k l}\left(r_{0}\right) \cos (l \varphi)\right] \\
& \cdot\left(\sum_{k=0}^{\infty} \sum_{l=0}^{\infty} R_{k l}\left(r_{0}\right)\left[\cos (l \varphi) \cdot C_{k l}+\sin (l \varphi) \cdot D_{k l}\right]-u\right)
\end{aligned}
$$

Suppose the solutions consist of a slowly varying component and a small-amplitude fast varying component, i.e.,

$$
\begin{aligned}
& \varphi(\tau)=\bar{\varphi}(\tau)+\frac{1}{R} \underline{\varphi}(R \tau) \\
& u(\tau)=\bar{u}(\tau)+\frac{1}{R} \underline{u}(R \tau) \\
& C_{k l}(\tau)=\overline{C_{k l}}(\tau)+\frac{1}{R} \underline{C_{k l}}(R \tau) \\
& D_{k l}(\tau)=\overline{D_{k l}}(\tau)+\frac{1}{R} \underline{D_{k l}}(R \tau)
\end{aligned}
$$

Substituting Eqs. (37)-(40) into Eqs. (33)-(36) results in,

$$
\begin{aligned}
& r_{0}\left[\left(\bar{\varphi}^{\prime \prime}+R \frac{\mathrm{d}^{2} \underline{\varphi}}{\mathrm{d}(R \tau)^{2}}\right)+2 \zeta_{1} \beta_{1}\left(\bar{\varphi}^{\prime}+\frac{\mathrm{d} \underline{\varphi}}{\mathrm{d}(R \tau)}\right)\right. \\
& \left.\quad+\left(\beta_{1}^{2}+\frac{1}{2} \beta_{3}^{2}\right)\left(\bar{\varphi}+\frac{1}{R} \underline{\varphi}\right)\right] \\
& \quad-\frac{1}{2} \beta_{3}^{2}\left(\bar{u}+\frac{1}{R} \underline{u}\right)=\mu \beta_{2}^{2}\left[w-\left(\bar{u}+\frac{1}{R} \underline{u}\right)\right] \\
& \quad+B_{\mathrm{d}} \sin (R \tau)
\end{aligned}
$$

$$
\begin{aligned}
& \left(\bar{u}^{\prime \prime}+R \frac{\mathrm{d}^{2} \underline{u}}{\mathrm{~d}(R \tau)^{2}}\right)+2 \zeta_{2} \beta_{2}\left(\bar{u}^{\prime}+\frac{\mathrm{d} \underline{u}}{\mathrm{~d}(R \tau)}\right) \\
& \quad+\beta_{2}^{2}\left(\bar{u}+\frac{1}{R} \underline{u}-w\right)-\frac{1}{2} \beta_{3}^{2} r_{0}\left(\bar{\varphi}+\frac{1}{R} \underline{\varphi}\right) \\
& \quad+\frac{1}{2} \beta_{3}^{2}\left(\bar{u}+\frac{1}{R} \underline{u}\right) \\
& =\frac{-F}{m \omega_{\mathrm{cr}}^{2}}
\end{aligned}
$$

$$
\begin{aligned}
& \left({\overline{C_{k l}}}^{\prime \prime}+R \frac{\mathrm{d}^{2} \underline{C_{k l}}}{\mathrm{~d}(R \tau)^{2}}\right)+2 l \bar{\Omega}\left({\overline{D_{k l}}}^{\prime}+\frac{\mathrm{d} \underline{D_{k l}}}{\mathrm{~d}(R \tau)}\right) \\
& +\left(\beta_{k l}^{2}-l^{2} \bar{\Omega}^{2}\right)\left(\overline{C_{k l}}+\frac{1}{R} \underline{C_{k l}}\right) \\
& =\gamma_{k l} \beta_{2}^{2}\left[-R_{k l}\left(r_{0}\right) \cos l\left(\bar{\varphi}+\frac{1}{R} \underline{\varphi}\right)\right. \\
& \left.-\frac{h}{2 r_{0}} \mu l R_{k l}\left(r_{0}\right) \sin l\left(\bar{\varphi}+\frac{1}{R} \underline{\varphi}\right)\right] \\
& \cdot\left(\sum_{k=0}^{\infty} \sum_{l=0}^{\infty} R_{k l}\left(r_{0}\right)\left[\cos l\left(\bar{\varphi}+\frac{1}{R} \underline{\varphi}\right) \cdot\left(\overline{C_{k l}}+\frac{1}{R} \frac{C_{k l}}{}\right)\right)\right. \\
& \left.\left.+\sin l\left(\bar{\varphi}+\frac{1}{R} \underline{\varphi}\right) \cdot\left(\overline{D_{k l}}+\frac{1}{R} \frac{D_{k l}}{}\right)\right]-\left(\bar{u}+\frac{1}{R} \underline{u}\right)\right) \\
& \left({\overline{D_{k l}}}^{\prime \prime}+R \frac{\mathrm{d}^{2} \underline{D_{k l}}}{\mathrm{~d}(R \tau)^{2}}\right)-2 l \bar{\Omega}\left({\overline{C_{k l}}}^{\prime}+\frac{\mathrm{d} \underline{C_{k l}}}{\mathrm{~d}(R \tau)}\right) \\
& +\left(\beta_{k l}^{2}-l^{2} \bar{\Omega}^{2}\right)\left(\overline{D_{k l}}+\frac{1}{R} \underline{D_{k l}}\right) \\
& =\gamma_{k l} \beta_{2}^{2}\left[-R_{k l}\left(r_{0}\right) \sin l\left(\bar{\varphi}+\frac{1}{R} \underline{\varphi}\right)\right. \\
& \left.+\frac{h}{2 r_{0}} \mu l R_{k l}\left(r_{0}\right) \cos l\left(\bar{\varphi}+\frac{1}{R} \underline{\varphi}\right)\right] \\
& \cdot\left(\sum _ { k = 0 } ^ { \infty } \sum _ { l = 0 } ^ { \infty } R _ { k l } ( r _ { 0 } ) \left[\cos l\left(\bar{\varphi}+\frac{1}{R} \underline{\varphi}\right)\right.\right. \\
& \left.\cdot\left(\overline{C_{k l}}+\frac{1}{R} \underline{C_{k l}}\right)+\sin l\left(\bar{\varphi}+\frac{1}{R} \underline{\varphi}\right) \cdot\left(\overline{D_{k l}}+\frac{1}{R} \underline{D_{k l}}\right)\right] \\
& \left.-\left(\bar{u}+\frac{1}{R} \underline{u}\right)\right)
\end{aligned}
$$

By assuming $B_{\mathrm{d}}=C_{\mathrm{d}} R$ and grouping the terms of Eqs. (41)-(44) into the coefficients of $R, 1, \frac{1}{R}$, the following equations can be obtained when $R \gg 1$,

$$
\begin{aligned}
r_{0} \frac{\mathrm{d}^{2} \underline{\varphi}}{\mathrm{d}(R \tau)^{2}}-C_{\mathrm{d}} \sin (R \tau) & =O\left(\frac{1}{R}\right), \frac{\mathrm{d}^{2} \underline{u}}{\mathrm{~d}(R \tau)^{2}} \\
& =O\left(\frac{1}{R}\right), \frac{\mathrm{d}^{2} \underline{C_{k l}}}{\mathrm{~d}(R \tau)^{2}} \\
& =O\left(\frac{1}{R}\right), \frac{\mathrm{d}^{2} \underline{D}_{k l}}{\mathrm{~d}(R \tau)^{2}}=O\left(\frac{1}{R}\right)
\end{aligned}
$$




$$
\begin{aligned}
& r_{0}\left[\bar{\varphi}^{\prime \prime}+2 \zeta_{1} \beta_{1}\left(\bar{\varphi}^{\prime}+\frac{\mathrm{d} \underline{\varphi}}{\mathrm{d}(R \tau)}\right)+\left(\beta_{1}^{2}+\frac{\beta_{3}^{2}}{2}\right) \bar{\varphi}\right] \\
& \quad-\frac{\beta_{3}^{2}}{2} \bar{u}-\mu\left(v_{\mathrm{r}}\right) \beta_{2}^{2}\left[\sum _ { k = 0 } ^ { \infty } \sum _ { l = 0 } ^ { \infty } R _ { k l } ( r _ { 0 } ) \left(\cos l\left(\bar{\varphi}+\frac{1}{R} \underline{\varphi}\right) \cdot \overline{C_{k l}}\right.\right. \\
& \left.\left.\quad+\sin l\left(\bar{\varphi}+\frac{1}{R} \underline{\varphi}\right) \cdot \overline{D_{k l}}\right)-\bar{u}\right]=O\left(\frac{1}{R}\right)
\end{aligned}
$$$$
\bar{u}^{\prime \prime}+2 \zeta_{2} \beta_{2}\left(\bar{u}^{\prime}+\frac{\mathrm{d} \underline{u}}{\mathrm{~d}(R \tau)}\right)
$$$$
+\beta_{2}^{2}\left[\bar{u}-\sum_{k=0}^{\infty} \sum_{l=0}^{\infty} R_{k l}\left(r_{0}\right)\left(\cos l\left(\bar{\varphi}+\frac{1}{R} \underline{\varphi}\right)\right.\right.
$$$$
\left.\left.\cdot \overline{C_{k l}}+\sin l\left(\bar{\varphi}+\frac{1}{R} \underline{\varphi}\right) \cdot \overline{D_{k l}}\right)\right]
$$$$
-\frac{1}{2} \beta_{3}^{2} r_{0} \bar{\varphi}+\frac{1}{2} \beta_{3}^{2} \bar{u}+\frac{F}{m \omega_{\mathrm{cr}}^{2}}=O\left(\frac{1}{R}\right)
$$

$$
\begin{gathered}
{\overline{C_{k l}}}^{\prime \prime}+2 l \bar{\Omega}\left({\overline{D_{k l}}}^{\prime}+\frac{\mathrm{d} \underline{D_{k l}}}{\mathrm{~d}(R \tau)}\right)+\left(\beta_{k l}^{2}-l^{2} \bar{\Omega}^{2}\right) \overline{C_{k l}} \\
-\gamma_{k l} \beta_{2}^{2}\left[-R_{k l}\left(r_{0}\right) \cos l\left(\bar{\varphi}+\frac{1}{R} \underline{\varphi}\right)\right. \\
\left.-\frac{h}{2 r_{0}} \mu\left(v_{\mathrm{r}}\right) l R_{k l}\left(r_{0}\right) \sin l\left(\bar{\varphi}+\frac{1}{R} \underline{\varphi}\right)\right] \\
.\left[\sum _ { k = 0 } ^ { \infty } \sum _ { l = 0 } ^ { \infty } R _ { k l } ( r _ { 0 } ) \left(\cos l\left(\bar{\varphi}+\frac{1}{R} \underline{\varphi}\right)\right.\right. \\
\left.\left.. \overline{C_{k l}}+\sin l\left(\bar{\varphi}+\frac{1}{R} \underline{\varphi}\right) \cdot \overline{D_{k l}}\right)-\bar{u}\right]=O\left(\frac{1}{R}\right)
\end{gathered}
$$

$$
\begin{aligned}
{\overline{D_{k l}}}^{\prime \prime}-2 l \bar{\Omega}\left({\overline{C_{k l}}}^{\prime}+\frac{\mathrm{d} \underline{C_{k l}}}{\mathrm{~d}(R \tau)}\right)+\left(\beta_{k l}^{2}-l^{2} \bar{\Omega}^{2}\right) \overline{D_{k l}} \\
-\gamma_{k l} \beta_{2}^{2}\left[-R_{k l}\left(r_{0}\right) \sin l\left(\bar{\varphi}+\frac{1}{R} \underline{\varphi}\right)\right. \\
\left.+\frac{h}{2 r_{0}} \mu\left(v_{\mathrm{r}}\right) l R_{k l}\left(r_{0}\right) \cos l\left(\bar{\varphi}+\frac{1}{R} \underline{\varphi}\right)\right] \\
\cdot\left[\sum _ { k = 0 } ^ { \infty } \sum _ { l = 0 } ^ { \infty } R _ { k l } ( r _ { 0 } ) \left(\cos l\left(\bar{\varphi}+\frac{1}{R} \underline{\varphi}\right)\right.\right. \\
\left.\left.\cdot \overline{C_{k l}}+\sin l\left(\bar{\varphi}+\frac{1}{R} \underline{\varphi}\right) \cdot \overline{D_{k l}}\right)-\bar{u}\right]=O\left(\frac{1}{R}\right)
\end{aligned}
$$

$$
\underline{C_{k l}}(R \tau)=C_{3} R \tau+O\left(\frac{1}{R}\right)
$$$$
\underline{D_{k l}}(R \tau)=C_{4} R \tau+O\left(\frac{1}{R}\right)
$$

where $C_{1}, C_{2}, C_{3}$ and $C_{4}$ are constants, and they should be zero as it is unlikely for the fast varying components to grow infinitely with time. By substituting Eqs. (50)-(53) into Eqs. (46)-(49) and applying a fast-time-average operator $\frac{1}{2 \pi} \int_{0}^{2 \pi}(*) \mathrm{d}(R \tau)$ to Eqs. (46)(49) as well as omitting the small quantity $O\left(\frac{1}{R}\right)$, the new differential equations with respect to the slowly varying components are,

$$
\begin{aligned}
& r_{0}\left[\bar{\varphi}^{\prime \prime}+2 \zeta_{1} \beta_{1} \bar{\varphi}^{\prime}+\left(\beta_{1}^{2}+\frac{1}{2} \beta_{3}^{2}\right) \bar{\varphi}\right]-\frac{1}{2} \beta_{3}^{2} \bar{u} \\
& \quad-\beta_{2}^{2} f\left(\bar{\varphi}^{\prime}\right) \\
& \quad \cdot\left[\sum_{k=0}^{\infty} \sum_{l=0}^{\infty}\left(\cos l \bar{\varphi} \cdot \overline{C_{k l}}+\sin l \bar{\varphi} \cdot \overline{D_{k l}}\right)-\bar{u}\right] \\
& \quad=0 \\
& \bar{u}^{\prime \prime}+2 \zeta_{2} \beta_{2} \bar{u}^{\prime} \\
& \quad+\beta_{2}^{2}\left[\bar{u}-\sum_{k=0}^{\infty} \sum_{l=0}^{\infty} R_{k l}\left(r_{0}\right)\left(\cos l \bar{\varphi} \cdot \overline{C_{k l}}+\sin l \bar{\varphi} \cdot \overline{D_{k l}}\right)\right] \\
& \quad-\frac{1}{2} \beta_{3}^{2} r_{0} \bar{\varphi}+\frac{1}{2} \beta_{3}^{2} \bar{u}+\frac{F}{m \omega_{\mathrm{cr}}^{2}} \\
& \quad=0
\end{aligned}
$$

$$
\begin{aligned}
{\overline{C_{k l}}}^{\prime \prime}+2 l \overline{\bar{\Omega}} \overline{D_{k l}}+\left(\beta_{k l}^{2}-l^{2} \bar{\Omega}^{2}\right) \overline{C_{k l}} \\
-\gamma_{k l}{ }_{2}^{2}\left[-R_{k l}\left(r_{0}\right) \cos l \bar{\varphi}-\frac{h}{2 r_{0}} l R_{k l}\left(r_{0}\right) f\left(\bar{\varphi}^{\prime}\right) \sin l \bar{\varphi}\right] \\
\cdot \quad\left[\sum_{k=0}^{\infty} \sum_{l=0}^{\infty} R_{k l}\left(r_{0}\right)\left(\cos l \bar{\varphi} \cdot \overline{C_{k l}}+\sin l \bar{\varphi} \cdot \overline{D_{k l}}\right)-\bar{u}\right]=0
\end{aligned}
$$

It can be derived from (45) that, 


$$
\begin{aligned}
{\overline{D_{k l}}}^{\prime \prime}-2 l \bar{\Omega}{\overline{C_{k l}}}^{\prime}+\left(\beta_{k l}^{2}-l^{2} \bar{\Omega}^{2}\right) \overline{D_{k l}} \\
-\gamma_{k l} \beta_{2}^{2}\left[-R_{k l}\left(r_{0}\right) \sin l \bar{\varphi}+\frac{h}{2 r_{0}} l R_{k l}\left(r_{0}\right) f\left(\bar{\varphi}^{\prime}\right) \cos l \bar{\varphi}\right] \\
\cdot\left[\sum_{k=0}^{\infty} \sum_{l=0}^{\infty} R_{k l}\left(r_{0}\right)\left(\cos l \bar{\varphi} \cdot \overline{C_{k l}}+\sin l \bar{\varphi} \cdot \overline{D_{k l}}\right)-\bar{u}\right]=0
\end{aligned}
$$

where

$f\left(\bar{\varphi}^{\prime}\right)=\frac{1}{2 \pi} \int_{0}^{2 \pi} \mu\left(\Omega-\omega_{\mathrm{cr}} \bar{\varphi}^{\prime}+\frac{\omega_{\mathrm{cr}} C_{\mathrm{d}}}{r_{0}} \cos (R \tau)\right) \mathrm{d}(R \tau) . \quad$ It is difficult to derive the analytical solution of the integral here, therefore the Gaussian quadrature is utilized to obtain the function values of $f\left(\bar{\varphi}^{\prime}\right)$. Furthermore, the derivative of $f\left(\bar{\varphi}^{\prime}\right)$ at $\bar{\varphi}^{\prime}=0$ can be obtained by the finite difference method. Subsequently Eqs. (54)-(57) can be linearized at the equilibrium point and the Jacobian matrix is extracted from the linearized system, whose eigenvalues indicate the stability of the system at the equilibrium point, i.e., if the self-excited vibration will happen, when the harmonic excitation is applied.

\section{References}

1. Berger EJ (2002) Friction modelling for dynamic system simulation. Appl Mech Rev 55(6):535-577

2. Popp K, Hinrichs N, Oestreich M (1995) Dynamical behaviour of a friction oscillator with simultaneous self and external excitation. Sadhana 20(2-4):627-654

3. Kinkaid NM, O'Reilly OM, Papadopoulos P (2003) Automotive disc brake squeal. J Sound Vib 267(1):105-166

4. Mills HR (1938) Brake squeak. Technical report 9000 B, Institution of Automobile Engineers

5. Popp K, Stelter P (1990) Stick-slip vibrations and chaos. Philos Trans R Soc A Math Phys Eng Sci 332(1624):89-105

6. Spurr RT (1961) A theory of brake squeal. Archive: Proc IMechE, Autom Div 1947-1970, 1961:33-52

7. North NR (1976) Disc brake squeal. Proc IMechE C 38(76):169-176

8. Popp K, Hinrichs N, Oestreich M (1996) Analysis of a selfexcited friction oscillator with external excitation. In: Guran A, Pfeiffer F, Popp K (eds) Dynamics with friction: modeling, analysis and experiment. World Scientific, Singapore, pp 1-35

9. Papangelo A, Ciavarella M, Hoffmann N (2017) Subcritical bifurcation in a self-excited single-degree-of-freedom system with velocity weakening-strengthening friction law: analytical results and comparison with experiments. Nonlinear Dyn 90(3):2037-2046

10. Tonazzi D, Massi F, Baillet L, Culla A, Di Bartolomeo M, Berthier Y (2015) Experimental and numerical analysis of frictional contact scenarios: from macro stick-slip to continuous sliding. Meccanica 50(3):649-664

11. Tucker RW, Wang C (2003) Torsional vibration control and cosserat dynamics of a drill-rig assembly. Meccanica 38(1):145-161

12. Hoffmann N, Gaul L (2004) A sufficient criterion for the onset of sprag-slip oscillations. Arch Appl Mech 73(9-10):650-660

13. Sinou JJ, Thouverez F, Jezequel L (2003) Analysis of friction and instability by the centre manifold theory for a nonlinear sprag-slip model. J Sound Vib 265(3):527-559

14. Hoffmann N, Fischer M, Allgaier R, Gaul L (2002) A minimal model for studying properties of the mode-coupling type instability in friction induced oscillations. Mech Res Commun 29(4):197-205

15. Hoffmann N, Gaul L (2003) Effects of damping on modecoupling instability in friction induced oscillations. Z Angew Math Mech 83(8):524-534

16. Kang J, Krousgrill CM, Sadeghi F (2008) Dynamic instability of a thin circular plate with friction interface and its application to disc brake squeal. J Sound Vib 316(1-5):164-179

17. Kinkaid NM, O'Reilly OM, Papadopoulos P (2005) On the transient dynamics of a multi-degree-of-freedom friction oscillator: a new mechanism for disc brake noise. J Sound Vib 287(4-5):901-917

18. Chan SN, Mottershead JE, Cartmell MP (1994) Parametric resonances at subcritical speeds in discs with rotating frictional loads. IMechE J Mech Eng Sci 208(6):417-425

19. Hochlenert D, Spelsberg-Korspeter G, Hagedorn P (2007) Friction induced vibrations in moving continua and their application to brake squeal. ASME J Appl Mech 74(3):542-549

20. Ouyang H, Mottershead JE (2005) Dynamic instability of an elastic disk under the action of a rotating friction couple. ASME J Appl Mech 71(6):753-758

21. Liu N, Ouyang H (2019) Friction-induced vibration of a slider on an elastic disc spinning at variable speeds. Nonlinear Dyn 98(1):39-60

22. Chen GX, Liu QY, Jin XS, Zhou ZR (2008) Stability analysis of a squealing vibration model with time delay. J Sound Vib 311(1-2):516-536

23. Butlin T, Woodhouse J (2009) Sensitivity of friction-induced vibration in idealised systems. J Sound Vib 319(1-2):182-198

24. Wang SK, Woodhouse $\mathbf{J}$ (2011) The frequency response of dynamic friction: a new view of sliding interfaces. J Mech Phys Solids 59(5):1020-1036

25. Dezi M, Forte P, Frendo F (2014) Motorcycle brake squeal: experimental and numerical investigation on a case study. Meccanica 49(4):1011-1021

26. Massi F, Giannini O, Baillet L (2006) Brake squeal as dynamic instability: an experimental investigation. J Acoust Soc Am 120(3):1388-1398 
27. Sinou JJ, Dereure O, Mazet GB, Thouverez F, Jezequel L (2006) Friction-induced vibration for an aircraft brake system-part 1: experimental approach and stability analysis. Int J Mech Sci 48(5):536-554

28. Triches M Jr, Gerges SNY, Jordan R (2004) Reduction of squeal noise from disc brake systems using constrained layer damping. J Braz Soc Mech Sci 26(3):340-348

29. Hammerström L, Jacobson S (2006) Surface modification of brake discs to reduce squeal problems. Wear 261(1):53-57

30. Wang DW, Mo JL, Ge XH, Ouyang H, Zhou ZR (2016) Disc surface modifications for enhanced performance against friction noise. Appl Surf Sci 382:101-110

31. Nakano K, Tadokoro C, Kado N (2013) Yawing angular misalignment provides positive damping to suppress frictional vibration: basic applicability to disc brake systems. SAE Int J Passenger Cars Mech Syst 6(2013-012069):1493-1498

32. Cunefare KA, Graf AJ (2002) Experimental active control of automotive disc brake rotor squeal using dither. J Sound Vib 250(4):579-590

33. Feeny BF, Moon FC (2000) Quenching stick-slip chaos with dither. J Sound Vib 237:173-180

34. Zhao X, Gräbner N, von Wagner U (2019) Avoiding creep groan: investigation on active suppression of stick-slip limit cycle vibrations in an automotive disk brake via piezoceramic actuators. J Sound Vib 441:174-186

35. Fidlin A (2005) Nonlinear oscillations in mechanical engineering. Springer, Berlin
36. Thomsen JJ (1999) Using fast vibrations to quench frictioninduced oscillations. J Sound Vib 228(5):1079-1102

37. Marques F, Flores P, Claro JP, Lankarani HM (2016) A survey and comparison of several friction force models for dynamic analysis of multibody mechanical systems. Nonlinear Dyn 86(3):1407-1443

38. Chung J, Oh JE, Yoo HH (2000) Non-linear vibration of a flexible spinning disc with angular acceleration. J Sound Vib 231(2):375-391

39. Van de Vrande BL, Van Campen DH, De Kraker A (1999) An approximate analysis of dry-friction-induced stick-slip vibrations by a smoothing procedure. Nonlinear Dyn 19(2):159-171

40. Chen L, Xi G (2014) Stability and response of a self-amplified braking system under velocity-dependent actuation force. Nonlinear Dyn 78(4):2459-2477

41. Li Z, Ouyang H, Guan Z (2016) Nonlinear friction-induced vibration of a slider-belt system. ASME J Vib Acoust 138(4):041006

42. Kang J, Krousgrill CM, Sadeghi F (2009) Comprehensive stability analysis of disc brake vibrations including gyroscopic, negative friction slope and mode-coupling mechanisms. J Sound Vib 324(1-2):387-407

Publisher's Note Springer Nature remains neutral with regard to jurisdictional claims in published maps and institutional affiliations. 